Universität Konstanz

Sozialwissenschaftliche Fakultät

Forschungsschwerpunkt »Gesellschaft und Familie»

Familienberichte:

Aufgabe, Probleme und Lösungsversuche der Sozialberichterstattung über die Familie

Kurt Lüscher

Arbeitspapier Nr.32

August 1999 
Kurt Lüscher ${ }^{1}$

\section{Familienberichte: Aufgabe, Probleme und Lösungsversuche der Sozial- berichterstattung über die Familie}

\section{Inhaltsverzeichnis}

Zusammenfassung

Summary

1. Einleitung

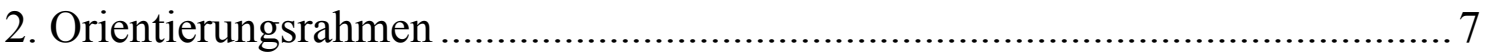

3. Beispiel: Der fünfte deutsche Familienbericht ............................................. 9

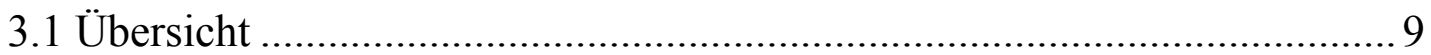

3.2 Die Stellungnahme der Bundesregierung .............................................12

3.3 Der Verlauf der Debatte im Bundestag ................................................14

3.4 Die Rezeption in der Tagespresse ............................................... 15

1 Der folgende Text beruht auf Arbeiten, die im Laufe der letzten Jahre in den Arbeitsbereichen "Familienpolitik" und "Familienrhetorik" am Konstanzer Forschungsschwerpunkt "Gesellschaft und Familie" durchgeführt worden sind, worauf im Folgenden an geeigneter Stelle im einzelnen hingewiesen wird. Ich danke insbesondere Regine Herbrik für die Mitarbeit bei der Auswertung der Pressedokumentation. Weitere Einsichten verdanke ich meiner Zugehörigkeit zum Wissenschaftlichen Beirat für Familienfragen beim (jetzigen) Bundesministerium für Familie, Senioren, Frauen und Jugend und der Mitarbeit an dessen Gutachten (siehe die Übersicht im Anhang). Ferner war ich an der Ausarbeitung des Berichtes "Familienpolitik in der Schweiz" (1982) beteiligt. F.X. Kaufmann und R. von Schweitzer haben mir ihre kritische Stellungnahme zu einer früheren Fassung des Textes zur Verfügung gestellt. Vorgesehen ist die Veröffentlichung in einer von W. Bien (Deutsches Jugendinstitut) herausgegebenen Reihe über Sozialberichterstattung. 
3.5 Die Rezeption in der Verbandspresse und in wissenschaftlichen Zeitschriften 18

4. Die Institutionalisierung der deutschen Familienberichte. .23

5. Entwicklungstendenzen vom ersten bis zum fünften Familienbericht .27

6. Familienberichte in anderen Ländern. 35

7. Ausblick 39

8. Literatur 42

9. Anhang .48

9.1 Eckdaten der Familienberichte der Bundesregierung 48

9.2 Gutachten des wissenschaftlichen Beirates für Familienfragen beim "Bundesfamilienministerium"

9.3 Inhaltsverzeichnisse der deutschen und der italienischen Familienberichte. .50

Forschungsschwerpunkt "Gesellschaft und Familie" 58 


\section{Zusammenfassung}

Die Sozialberichterstattung über die Familie hat in Deutschland ebenso wie in anderen europäischen Ländern einen besonderen Ausdruck in der Form der sogenannten „Familienberichte" gebunden. Diese zeichnen sich in Deutschland durch eine „dialogische" Struktur aus: Sie bestehen aus der Darstellung eines unabhängigen Sachverständigengremiums und aus einer sich darauf beziehenden Stellungnahme der Bundesregierung.

Da diese Berichte gemäß gesetzlichem Auftrag dem Parlament regelmäßig vorgelegt werden müssen, weisen sie einen hohen Grad „manifester" Institutionalisierung auf. Dazu kommt eine „latente" Institutionalisierung, die sich aus einer engen thematischen und personellen Verpflichtung mit dem Wissenschaftlichen Beirat beim Familienministerium ergibt.

Die Rezeption der Berichte läßt sich in drei Phasen unterteilen: Die Diskussion im Parlament und in der Presse unmittelbar nach Erscheinen als Tagesereignis, die Rezension in der Fachpresse und die allgemeine Nutzung der Berichte im universitären Unterricht sowie in der Verwaltungsarbeit.

So weit wie möglich wird dies am Beispiel des fünften Familienberichtes dargestellt. Dieser wird überdies hinsichtlich der zentralen Themen, namentlich der zunehmenden Öffnung des Begriffes der Familie in die Reihe der bisher erschienenen Berichte eingeordnet.

Um die Besonderheiten, die Stärken und Schwächen der deutschen Familienberichte herauszuarbeiten, werden diese kurz mit jenen in anderen europäischen Ländern verglichen. Die Darstellung stellt insgesamt einen Versuch dar, die Familienberichterstattung konzeptuell mit den Mitteln eines interaktionistisch fundierten Ansatzes der Diskursanalyse zu erfassen. 


\section{Summary}

In Germany as well as in other European Countries a special means of social reporting on the family has been developed, namely the so-called „Family Reports". Especially in Germany these are characterized by a dialogical structure. They consist of an analysis by an independent group of experts, reactions and a position statement by the government.

Since the law requires that these reports be regularly submitted to parliament, they display a high degree of "manifest" institutionalization. This is supplemented by a "latent" institutionalization based on a close thematic and personnel connection with the Scientific Advisory Board of the Family Ministry.

The reception of the reports can be devided in three phases: A discussion in parliament and in the media immediately following publication, reception in journals and the general use of the reports in university teaching and in administrative work. As far as possible this reception is documented using the example of the fifth German Family Report. This report is also interpreted in terms of a few dominant topics and especially in view of broadening the definition of the family in the five German Family Reports.

In order to point out special features, the strength and weaknesses of the Germany Family Reports, they are compared with those of other European countries. The analysis itself is an attempt to view the social reporting of the family in the perspective of a discourse analysis based upon interactional premises. 


\section{Einleitung}

Unter die Bezeichnung "Sozialberichterstattung über die Familie" in einem weiten Sinne des Wortes fallen alle Veröffentlichungen, die systematisch gesellschaftliche Daten über die Entwicklung familialer Lebensformen und privater Haushalte präsentieren und kommentieren; dazu können Darstellungen familienpolitischer Maßnahmen und ihre Entwicklung sowie rechtliche Regelungen kommen. Dabei gibt es Überschneidungen mit anderen Bereichen der Sozialberichterstattung, so über Kinder, Jugendliche, Frauen und ältere Menschen oder über das Gesundheitswesen. Publikationen dieser Art gibt es für viele einzelne Länder und auch auf internationaler Ebene. ${ }^{2}$ In einem engeren Sinne versteht man darunter "Familienberichte", die regelmäßig von wissenschaftlichen Kommissionen oder Forschungseinrichtungen erstellt werden. Es gibt sie in Deutschland, auch in einzelnen Bundesländern, ferner in Österreich, Italien und in der Schweiz.

Diese "Familienberichte", die in der Regel einen offiziellen oder zumindest einen offiziösen Status haben, stehen im Folgenden im Zentrum meiner Darstellung. An ihrem Beispiel können nämlich eine Reihe von theoretisch und forschungspraktisch interessanten Fragen erörtert werden. Sie hängen bis zu einem gewissen Grad mit Besonderheiten von Familie zusammen, namentlich der Offenheit des Begriffs "Familie", der einer gewissen Offenheit der Institutionalisierung entspricht. Sie ist - jedenfalls was die rechtlichen Regelungen betrifft - größer ist als diejenige der Ehe. Im weiteren unterstreicht die widersprüchliche Dynamik in der Entwicklung familialer Lebensformen, zieht man die alltägliche Praxis in Betracht, die Aktualität der Thematik.

2 Einen Beitrag zur Bestandsaufnahme in Europa hat unlängst Rothenbacher (1997) in dem von Noll (1997) herausgegebenen Sammelband über Sozialberichterstattung publiziert, wobei allerdings für die Schweiz der weiter hinten erwähnte Bericht von 1982 hinzuzufügen wäre. - Zur europäischen Perspektive siehe auch Commaiile/De Singly (1997). 
Schließlich geht es nicht - wie vielfach bei der Sozialberichterstattung - um die Lebensform einer Minderheit. Indessen wird die gesellschaftliche Situation von Familie zusehends als problematisch empfunden. Strittig ist überdies, ob das Konzept des Risikos, das in anderen Bereichen der Sozialberichterstattung, z.B. über Armut und Gesundheit, von Belang ist, auf Familie anwendbar ist.

Trotz oder vielleicht gerade wegen dieser Besonderheiten verweisen die Familienberichte auf Fragen konzeptueller Art, die für die Sozialberichterstattung insgesamt von Belang sind. Bezugspunkt meiner Darstellung sind die nationalen deutschen Familienberichte, weil diese - wie ich zeigen werde - in Verbindung mit einem hohen Grad der Institutionalisierung eine Reihe theoretisch und praktisch beachtenswerter Merkmale aufweisen.

\section{Orientierungsrahmen}

In meinem Verständnis - und damit der Orientierungsrahmen in den Horizont, der meiner Darstellung zugrunde liegt - kann man diese Familienberichte (also die Familienberichterstattung im engeren Sinne des Wortes) in Anlehnung an ein auf W. I. Thomas zurückgehendes soziologisches Konzept auffassen, nämlich als Versuche einer "Definition der (gesellschaftlichen) Situation" von Familie. Es geht also darum, zu einem bestimmten Zeitpunkt und in einem gesellschaftlichen Kontext (einem Nationalstaat, einem Land, einer Kommune, Europa) zu umschreiben, was mit "Familie" gemeint ist und wie Familie gelebt wird, auch was damit gemeint sein soll. Überdies geht es darum, welche Bedingungen zu schaffen sind, damit Familie "richtig" gelebt werden könnte, und wie eine derartige "Familienpolitik" begründet werden kann. 
Das bedingt meiner Auffassung nach ausdrücklich oder stillschweigend die Befassung mit der Frage der "Definition" bzw. der Leitbilder von Familie. ${ }^{3}$ Das ist insbesondere notwendig, wenn ausführlich auf die Begründung und die Ausgestaltung familienpolitischer Maßnahmen und Einrichtungen eingegangen wird. Mit guten Gründen kann man nämlich die allgemeine These vertreten, familienpolitische Maßnahmen, Einrichtungen und Programme würden eine Familiendefinition voraussetzen, diese bekräftigen oder verändern. Dabei geht es in der Regel um mehr als nur nominelle oder operationale Definitionen. Wenn öffentlich von Familie die Rede ist, sind nämlich meistens ethische, moralische und ordnungspolitische Aspekte im Spiel. Ich möchte dies die These der Korrespondenz von Familiendefinition und Familienpolitik nennen.

Im Anschluß daran könnte man etwas zugespitzt sagen: Wir können nicht nicht politisch und moralisch über Familie reden und schreiben; jedenfalls können wir nicht verhindern, daß das Reden und Schreiben über Familie solchermaßen aufgefaßt wird. Wenn dem so ist - und ich gehe im Folgenden von dieser Behauptung aus - dann werden wir darauf verwiesen, daß es immer auch um Überzeugungen geht, was richtigerweise mit Familie gemeint ist, wie Familie sozial gestaltet werden soll. So gesehen sind Familienberichte ein Teil der "Familienrhetorik". ${ }^{4}$ Eine zusätzliche theoretische Brisanz ergibt sich aus dem Umstand, daß die Familienberichte selbst Teil des Kataloges familienpolitischer

3 Für mein Verständnis des Konzeptes des Leitbildes siehe Lüscher 1997. - Es gehört zu den bemerkenswerten Besonderheiten der Thematik, dass die "Definition" von Familie auch in den Wissenschaften immer wieder Anlass zu Kontroversen bietet. Darin wird eine normative Komponente von Definitionen erkennbar, die praxis- und politikrelevanten Sachverhalten eigen ist. Eine Möglichkeit des Umgangs mit dieser Frage besteht darin, die normativen Bezüge als solche in die Definition miteinzubeziehen, also zu sagen: Der Begriff der Familie (als soziale Kategorie) bezeichnet in der Gegenwart westlicher Industriegesellschaften die primär durch die Gestaltung der Beziehungen zwischen Eltern und Kindern sowie der Eltern untereinander konstituierten Lebensformen eigener Art, die als solche gesellschaftlich anerkannt (legitimiert) werden.

4 Für das Verständnis dieses Konzeptes siehe Lüscher 1995a, seine Anwendungen auf die Familiensoziologie: Bräuninger et al. 1996. Eine Artikelfassung ist zur Publikation angenommen (Lange et al. in Vorbereitung). 
Maßnahmen und Einrichtungen sind. ${ }^{5}$ Zur Diskussion steht somit auch, inwiefern es angemessen und wissenschaftlich ertragreich ist, Sozialberichterstattung unter Gesichtspunkten der Diskurs- und Rhetorikanalyse zu betrachten, und welche Folgerungen sich daraus für den wissenschaftlichen Stellenwert der Berichte ergeben. Systematisch betrachtet geht es um eine Analyse der Verknüpfung von Inhalten, Akteuren, Rezeption und sozialen Kontexten der Sozialberichterstattung.

\section{Beispiel: Der fünfte deutsche Familienbericht}

\section{1 Übersicht}

Der Fünfte Familienbericht von 1995 wurde von einer aus sieben Mitgliedern bestehenden Sachverständigenkommission erstellt. Er umfaßt 342 Druckseiten; ihm ist eine 34-seitige Stellungnahme der Bundesregierung vorausgeschickt. Die Stellungnahme wurde unter Federführung des zuständigen Ministeriums und in Konsultation mit anderen Ministerien erstellt. Im Rahmen ihrer Arbeiten ließ die Kommission 19 Expertisen erarbeiten, die als "Materialien" ebenfalls veröffentlicht worden sind. Das Kommissionssekretariat besorgte mit drei wissenschaftlichen Mitarbeiterinnen und Mitarbeitern das DJI. - Es ist hier nicht der Ort, ausführlich auf den Inhalt des Berichtes einzugehen. Wichtig ist unter allgemeinen Gesichtspunkten der Sozialberichterstattung das Folgende:

- Es handelt sich um den ersten Bericht nach der Vereinigung der in der Nachkriegsepoche entstandenen deutschen Staaten. Dieser Aspekt kommt durchgängig zur Sprache. Er ist besonders markant im Kapitel "Familienrecht", das in den Vorschlag einmündet, "die Möglichkeiten einer in sich geschlossenen

5 Das Beispiel der Schweiz, wo im Bericht von 1982 ausdrücklich eine institutionalisierte Familienberichter-stattung gefordert wurde, die Bundesbehörden jedoch der Empfehlung nicht folgen mochten, zeigt diesen Zusammenhang. 
Kodifikation des Familienrechtes zu prüfen. Das würde bedeuten, das Familienrecht im Ganzen neu zu konzipieren und zu regeln." (V: 102) ${ }^{6}$

- Der Bericht wurde vom Bemühen getragen, eine neuartige konzeptuelltheoretische Begründung für Familienpolitik vorzulegen. Ihr Kern liegt in der These, "daß das Humanvermögen einer Gesellschaft durch die Leistungen der Familie begründet wird, und alle Menschen von Lebensbeginn bis zum Lebensende sowie die Gesamtheit der gesellschaftlichen Einrichtungen dieser familialen Leistungen bedürfen" (V: 16) ${ }^{7}$.

Das Konzept des Humanvermögens ist in zweifacher Hinsicht doppeldeutig: Zum einen werden darin sowohl ökonomische als auch soziale Dimensionen (Potentiale und Leistungen) angesprochen. Überdies ist sowohl das "Gesamtpotential der Kompetenzen der Mitglieder einer Gesellschaft gemeint ebenso wie das Handlungspotential des Einzelnen" (V: 28). - Diese Mehrdeutigkeit festzustellen ist auch unter Bezug auf meine allgemeine Charakterisierung der "Situationsdefinition" wichtig. Der offene diskursive Charakter des Berichtes wird dadurch bekräftigt..

Komplementär $\mathrm{zu}$ diesem Konzept ist dasjenige der "strukturellen Rücksichtslosigkeit" zu verstehen. Es beinhaltet eine Kritik an der im einzelnen in den verschiedensten Lebensbereichen aufzeigbaren geringen Wertschätzung und der Mißachtung der Belange von Familie, die sich in erheblichen sozialen und wirtschaftlichen Benachteiligungen ausdrücken. Der Begriff der "strukturellen Rücksichtslosigkeit" ist ein wichtiges Element der "Rhetorik" des

$6 \quad$ Fünfter Familienbericht, S. 102. - Hier und im Folgenden bezeichnet die römische Ziffer den Familienbericht, die arabische die Seitenzahl.

7 Für eine ausführliche Darstellung des Begriffes des Humanvermögens, der nicht mit demjenigen des "Humankapitals" identisch ist, siehe auch Krüsselberg 1997, Kapitel II., Teile 4 und 5; Lampert 1993; Lampert 1996: 28-41. 
Berichtes. $^{8}$ Dieser Linie entspricht im weiteren, daß die traditionelle Kennzeichnung von familienpolitischen Transferleistungen als "Familienlastenausgleich" rhetorisch in einen "Familienleistungsausgleich" umformuliert wird. ${ }^{9}$

Unter formalen Gesichtspunkten ist die Zweiteilung des Dokuments hervorzuheben. Weitaus den größten Teil (342 Seiten) macht der Bericht der Sachverständigenkommission aus. Diesem in der publizierten Fassung vorangestellt ist die "Stellungnahme der Bundesregierung" (XXXIV Seiten). Hier zeigt sich der auf Diskurs angelegte Charakter. Die Regierung ist der erste Adressat des eigentlichen Berichtes und befaßt sich mit ihm schon vor seiner Veröffentlichung. Diese Stellungnahme wird in einer langen Reihe von Beratungen zwischen den Ministerien und Abteilungen unter Federführung des Familienministeriums erstellt.

Nach der Veröffentlichung sind in einer ersten Phase das Parlament (als Auftraggeber) und die Medien die Adressaten. In einer zweiten Phase der Rezeption, die vergleichsweise offen ist, setzt die Auseinandersetzung seitens der Fachleute ein. Wenig ist bekannt, ob und wie das in der Verwaltung geschieht. Seitens der Familienverbände liegen einige Kommentare zum Familienbericht vor. Im weiteren gibt es Rezensionen und kritische Auseinandersetzungen in den

8 Der Begriff wurde - so weit ich sehe - erstmals von Kaufmann (1990) in seiner gutachterlichen Charakter aufweisenden Publikation in der Schriftenreihe des Bundeskanzleramtes veröffentlicht, also ebenfalls im Umkreis von "Sozialberichterstattung".

9 Allerdings sind nicht alle Transfers, die bisher unter den Begriff des Lastenausgleichs subsumiert wurden, als Leistungsausgleich zu interpretieren. Die Frage, wie die spezifischen Leistungen von Familie sozialpolitisch erfasst werden können, bedarf einer differenzierten Beantwortung. Das gleiche gilt hinsichtlich des Verhältnisses von Familienpolitik und Steuerpolitik. Diese Thematik hat im Gefolge der Beschlüsse des Bundesverfassungsgerichtes vom 10.11.1998 an Relevanz (und Brisanz) gewonnen. 
wissenschaftlichen Fachzeitschriften. ${ }^{10}$ Eine - allerdings nicht klar umschreibbare - dritte Phase kann man in der Nutzung der Berichte im Unterricht an (Fach)Hochschulen und in der Verwaltung sehen.

\subsection{Die Stellungnahme der Bundesregierung}

In dieser Stellungnahme kommt über weite Strecken Zustimmung zum Kommissionsbericht zum Ausdruck, so hinsichtlich des Bemühens, von einem Familienverständnis auszugehen, "das sich an der Lebenswirklichkeit mit unterschiedlichen Familienformen orientiert" (V: IV), sowie hinsichtlich der Auffassung, "daß die Gesellschaft einer grundlegenden Umorientierung hin zu mehr Familienfreundlichkeit bedarf" (V: VI), und es "ein besonderes Anliegen der Bundesregierung ist, die im Familienbericht thematisierten strukturellen Rücksichtslosigkeiten gegenüber Familien abzubauen". (V: VI) ${ }^{11}$

Zustimmung findet die Auffassung, "daß junge Eltern stärker als bisher in ihren Erziehungsaufgaben zu unterstützen sind"; hier erfolgt ein Verweis auf das KJHG. Im weiteren wird örtliche und regional gestaltete Familienpolitik als Schwerpunkt zustimmend hervorgehoben, da, wie es heißt, die verschiedenen politischen Ebenen in einem sich ergänzenden Verhältnis stehen. Auch die Ausführungen über eine bessere Vereinbarkeit von Familie und Beruf finden Akzeptanz, ebenso alles in allem die Stellungnahmen zu den Kapiteln "Bildung" und "Gesundheit". ${ }^{12}$

10 Siehe hierzu z.B. auch das Besprechungsforum des 2. Familienberichts in der ZfS 1976: Frühere Berichte waren auch Gegenstand von Beratungen bei Kongressen der Deutschen Gesellschaft für Soziologie.

11 Hier bezeichnen die römischen Seitenzahlen die originale Paginierung der "Stellungnahme".

12 Stefan/Keil (1994) bemerken, dass insbesondere jene Bereiche Zustimmung fanden, in denen kommunale und länderspezifische Kompetenzen und Verpflichtungen der Familienpolitik angesprochen werden, während die Stellungnahme auf Bundesebene eher rechtfertigend ausfällt (siehe auch weiter hinten). 
Hingegen will sich die Bundesregierung die Annahmen, die der Berechnung des Geldwertes familialer Leistungen zugrunde liegen, "im einzelnen nicht zu eigen machen" (V: VI). Ebensowenig teilt sie die Empfehlungen zur Reform des Familienlastenausgleichs, insbesondere mittels Reform der Ehegattenbesteuerung. Hier wirft sie die Frage der Verfassungsgemäßheit auf. Akzentuiert zurückgewiesen wird das Kapitel "Familienrecht", wo eine "stark wertende Darstellung" geortet wird, "die nach Auffassung der Bundesregierung weder der vom BGB gestalteten freiheitlichen Rechtsordnung einerseits noch der Lebenswirklichkeit in der DDR andererseits gerecht zu werden vermag." (V: $\mathrm{XV})$

Die Stellungnahme schließt - unter Bezug auf das Internationale Jahr der Familie - mit einem allgemeinen Bekenntnis. Es wird gesagt, die Familie habe nach wie vor einen hohen Stellenwert. Die Menschen wollten in Familie leben, junge Menschen wollten Kinder, wobei die meisten von ihnen auch heiraten wollten, "viele allerdings erst, wenn die Entscheidung für ein Kind oder mehrere Kinder gefallen ist." (V: XXIII) Gleichzeitig sei unbestritten, daß Familie unverzichtbar und ohne Alternative sei:

"- Als Ort, wo Kinder geboren werden und zu lebenstüchtigen, verantwortungsbewußten Bürgern heranwachsen können,

- als Ort, der dauerhafte und verläßliche Beziehungen von Menschen ermöglicht und sichert und damit emotionale Geborgenheit, Anerkennung und Mitmenschlichkeit finden hilft, und - als Ort der Solidarität der Generationen." (ebd.)

Darin drückt sich selbstverständlich eine markante politische, sogar weltanschauliche Überzeugung aus. Es handelt sich also um eine betont rhetorische Passage. Das zeigt sich auch darin, daß ähnliche Formulierungen in ministerialen Verlautbarungen immer wieder vorkommen. Sie haben die Form 
präskriptiver Deskriptionen. Dabei tritt die Tatsache der Pluralität familialer Lebensformen in bemerkenswerter Weise in den Hintergrund..

\subsection{Der Verlauf der Debatte im Bundestag}

Liest man den Verlauf der Debatte im Bundestag nach, so fällt auf, wie wenig auf den Inhalt des Berichtes Bezug genommen wird. ${ }^{13}$ Jedenfalls wird an keiner Stelle auf explizite Empfehlungen eingegangen, wobei allerdings festzuhalten ist, daß die Sachverständigenkommission darauf verzichtet hat, einen eigentlichen Empfehlungskatalog aufzustellen und an prominenter Stelle zu plazieren (wie das in vorhergehenden Familienberichten der Fall gewesen ist). Der verhaltene Bezug auf den Bericht hat möglicherweise seinen Grund in dem Umstand, daß wie in einem Votum ausdrücklich beanstandet wird - den Abgeordneten der Bericht anscheinend erst am Vorabend der Debatte zugestellt worden ist. Die Presseorientierung fand allerdings eine Woche vorher statt. ${ }^{14}$ Wie dem auch sei: Eine gewisse Eile bestand, den Bericht kurz vor Ende der Legislaturperiode zu debattieren. Dementsprechend wurden die meisten Voten benutzt, um die von der Regierung und der Opposition vertretenen Positionen darzustellen bzw. wechselseitig zu kritisieren.

Es ging vor allem um die Ausgestaltung des Familienlastenausgleichs. Die im Bericht enthaltene Verschiebung der Terminologie zum Familienleistungsausgleich wird von Regierung und Opposition vereinzelt, allerdings kommentarlos, übernommen. Die Ministerin ihrerseits betonte eingangs pauschal, daß - erstens - Menschen in Familien leben wollten, daß zweitens - die große Mehrheit junger Menschen Kinder wollten und daß drittens - unsere Gesellschaft Familie brauche, die Familie allerdings unter

13 Deutscher Bundestag, 12. Wahlperiode, 236. Sitzung, Freitag, den 24. Juni 1994, S. 2071820737. 
Wettbewerbsnachteilen und 'strukturellen Rücksichtslosigkeiten' leide. Sie bediente sich also jener präskriptiv-deskriptiven rhetorischen Figuren, auf die ich bereits hingewiesen habe.

In einem einzigen Votum (Ursula Männle) wird das zentrale Konzept des "Humanvermögens" sowie die Vorstellung der Vielfalt von Familienformen grundsätzlich angesprochen. Doch auch dieser Beitrag kommt nicht ohne Beschwörung der Dauerhaftigkeit von Familie aus, was auf Assoziationen zu einem traditionellen Familienbild verweist. Kennzeichnend dafür ist das vorgelesene Zitat: "Die Familie lebt, ist in allen Wandlungen wiederauffindbar ...". - Schließlich stellt, nach einem Vergleich der Entwicklung im Osten und Westen, die ostdeutsche Abgeordnete Angelika Pfeiffer - sich an die Opposition wendend - fest: "Es geht den Familien bei allen Schwierigkeiten nicht so schlecht, wie Sie uns einreden wollen." (S. 20736)

\subsection{Die Rezeption in der Tagespresse ${ }^{15}$}

Die Dokumentation des Pressedienstes Argus umfaßt für die Zeit vom 16. Juni bis 30. Juli 1995 insgesamt 184 Artikel (bis zum 23.6.: 172 Artikel). Sie sind in 108 publizistischen Einheiten und - unter Berücksichtigung der Regionalausgaben - in 375 Zeitungen (inkl. Regionalausgaben) mit einer Gesamtauflage von rund 23,47 Millionen Exemplaren erschienen. Das entspricht annähernd der von Schütz (1998) ermittelten Gesamtauflage.

14 Hier stellt sich die Frage, warum die Abgeordneten nicht zumindest gleichzeitig mit den Medien den Bericht erhalten haben.

15 Bemühungen um eine differenzierte inhaltsanalytische Aufarbeitung der Materialien erwiesen sich als wenig ergiebig. Das hängt u.a. mit der Kürze der Artikel und den zahlreichen Wiederabdrucken zusammen. Häufig werden lediglich die Titel variiert. Ich beschränke mich darum im Folgenden auf eine zusammenfassende Wiedergabe unserer (quantitativen) Analysen. 
Rund zwei Drittel der Artikel stammen von Agenturen. Eine geringe Zahl ist nicht näher gekennzeichnet. Hierbei dürfte es sich ebenfalls um (gekürzte) Agenturberichte handeln. Gut ein Viertel hat einen namentlich erwähnten Verfasser oder Verfasserin, wobei hier wiederum viele mehrfach vorkommen. Man kann dementsprechend vermuten, daß sich nur einige wenige Journalistinnen und Journalisten vertieft für die Belange der Familie und der Familienpolitik interessieren.

Von den insgesamt 184 Artikeln sind weitaus die meisten am Tag nach der Medienorientierung erschienen. Über die Debatte im Bundestag haben nur etwa 15\% der Zeitungen mit einem speziellen hervorgehobenen Beitrag berichtet. Ich beschränke mich deshalb an dieser Stelle auf die Berichte über die Medienkonferenz und darauf, wie der Bericht angekündigt worden ist, also auf die Titelung. Eine gewichtete Auszählung, bei der die Auflagenhöhe der publizistischen Einheiten berücksichtigt wird, zeigt zunächst folgendes Bild:

Weitaus am häufigsten (Gesamtauflage 20 Millionen) werden familienpolitische Maßnahmen und Forderungen hervorgehoben, also beispielsweise:

- Was Bonn den Familien verspricht. Anspruch auf Kindergartenplatz und Teilzeitarbeit. (Abendzeitung) - Bei der Vorlage des ersten gesamtdeutschen Familienberichts: Politisches Schattendasein von Familienthemen beklagt. (Holsteinischer Kurier)

In vielen Titeln (Gesamtauflage rund 10 Millionen Exemplare) wird die Ministerin erwähnt, die Botschaft also personifiziert:

- Rönsch fordert mehr Geld für Familien mit Kindern. (Leipziger Volkszeitung) Rönsch bleibt dabei: Familie braucht mehr Unterstützung. Ministerin hält 
Zukunftsbeitrag der Kinderlosen in der Diskussion. (Aachener Volkszeitung) Hier ist die Verknüpfung mit einem parteispezifischen Vorschlag beachtenswert.

Annähernd gleich häufig wird auf den Familienbericht als solchen hingewiesen. Ebenfalls beliebt sind Titel, in denen demoskopische oder demographische Angaben gemacht werden, wobei die grundsätzlich positive Einstellung zu Ehe und Familie hervorgehoben wird, meist jedoch in Verbindung mit der kontrastierenden Feststellung, daß die Zahl der Einzelkinder zunehme, was ja eine objektiv nicht haltbare Behauptung darstellt. Dem positiv bewerteten Umstand, daß immer noch eine relativ große Anzahl von Menschen in Familien mit Kindern lebt, wird ein Trend zur freiwilligen Kinderlosigkeit von Paaren entgegengehalten.

- Jeder zweite lebt in Familie mit Kindern. Regierungsbericht über die Lage der Familie: Immer mehr deutsche Frauen bleiben kinderlos. (Westdeutsche Zeitung) - Ehe und Familie sind beliebteste Lebensformen. Zahl der Einzelkinder hat zugenommen. (Westfalenpost) - Bei Bild (Hamburg) verkürzt sich alles auf eine kleine Notiz: Zahl des Tages 809 000. (Damit ist die Zahl der 1992 Geborenen gemeint, die im Familienbericht genannt wird.)

Man gewinnt also den Eindruck, daß der Familienbericht als Aufhänger genutzt wird, um über Forderungen nach einem Ausbau der Familienpolitk, die insbesondere von der Ministerin erhoben werden, zu berichten, wobei besonders häufig das Postulat einer "Familienverträglichkeitsprüfung" ausdrücklich erwähnt wird, ferner kommt auch die Idee des "Zukunftsbeitrags der Kinderlosen" zur Sprache. Wenn die Familienpolitik als solche angesprochen wird, steht die Forderung nach Abbau von Wettbewerbsnachteilen der Familien im Vordergrund; ebenfalls häufig findet sich in den Titeln die Feststellung, die 
Leistung der Familien würde nicht ausreichend gewürdigt und Familienthemen führten in der Politik ein Schattendasein.

Zum Bericht selbst kann man schließlich lesen:

- Langweilig formuliert - doch voller Sprengstoff. Familienbericht der Bundesregierung ist keine Lobeshymne für die Koalition. (Südkurier)

Indessen wird er als erste gesamtdeutsche Darstellung der Familie gewürdigt. $\mathrm{Da}$ es sich im wesentlichen um die Analyse einer Sachverständigenkommission handelt, wird - was realistischerweise auch nicht erwartet werden kann - in der Titelung kaum je angesprochen; ebensowenig wird auf die duale Form des Berichts hingewiesen.

\subsection{Die Rezeption in der Verbandspresse und in wissenschaftlichen Zeitschriften}

Diese Rezeption ist schwierig zu dokumentieren. Was mir vorliegt, sind insgesamt 7 Besprechungen in Verbandszeitschriften sowie in wissenschaftlichen Fachzeitschriften. Im Grundtenor sind sie positiv und die rhetorischen Schlüsselbegriffe werden aufgenommen. Nur in einem Beitrag werden Sachverständigenbericht und Stellungnahme auseinandergehalten und miteinander verglichen. Im Folgenden gehe ich auf einige unter Gesichtspunkten der Sozialberichterstattung wesentlichen Punkte ein.

Bertram (1995) nimmt im Titel das Konzept des Humanvermögens auf und attestiert der Kommission, mit ihrem Versuch der Begründung von Familienpolitik theoretisches Neuland betreten zu haben. In einem durchgängig zustimmenden Duktus stellt er insbesondere einen Zusammenhang zwischen der "strukturellen Rücksichtslosigkeit" und der Entscheidung für Kinder her. Eigentlich sei es "außerordentlich erstaunlich ..., wenn sich noch junge 
Erwachsene und vor allem junge Frauen für Familie mit Kindern entscheiden" $(557) .{ }^{16}$

Die Forderungen des Berichtes lassen sich seiner Ansicht nach aber nur dann realisieren, "wenn es endlich gelingt, die falsche Zuordnung, daß Leistungen der Eltern für ihre Kinder als Privatangelegenheiten der Eltern interpretiert werden, aufzubrechen und zu akzeptieren, daß die Leistungen der Familie die Basis der Zukunft der ganzen Gesellschaft sind. Die familialen Investitionen in Menschen entscheiden nicht nur über die Wirtschaftspotentiale von morgen, sondern zugleich über die Kultur des menschlichen Zusammenlebens. Solange dies nicht in das öffentliche und politische Bewußtsein eingedrungen ist und das Handeln der politischen Parteien beeinflußt, so lange werden wir noch viele vorzügliche, mit Fachkompetenz geschriebene Familienberichte lesen können, die letztlich in ihrer Wirksamkeit daran scheitern müssen, daß Familienpolitik bis heute nicht als Strukturpolitik der Gesellschaft begriffen wird." (563) - Bemerkenswert ist hier, daß seitens eines Wissenschaftlers Zustimmung zu den politischen Positionen eines Familienberichts signalisiert wird und gleichzeitig - auf diffuse Weise - die Wirksamkeit solcher Berichte abhängig gemacht wird von einer fundamentalen Gesinnungsänderung, die doch eigentlich gerade durch diese Berichte in Gang gebracht werden sollte.

Stefan/Keil (1994) fassen den Bericht unter Wiedergabe der wichtigen Thesen und Daten zusammen und kommentieren ihn in einem zustimmenden Grundton. Kritisch äußern sie sich zur Stellungnahme der Regierung, namentlich wo die Situation beschönigt und das Regierungshandeln legitimiert wird. Das sei - vor allem in Wahlkampfzeiten - verständlich, bleibe aber "als Stellungnahme zu einem nicht hoch genug zu bewertenden wissenschaftlichen Bericht zur Lage der Familien unbefriedigend. Während dem Bericht selbst, in Sonderheit in seinen

16 Die Zahlen in Klammern bezeichnen die Seite im jeweils besprochenen Aufsatz. 
hochdifferenzierten analytischen Abschnitten, in Öffentlichkeit, Schule und Hochschulen in den nächsten Jahren ein intensiver Gebrauch als vorbildliches familienwissenschaftliches Nachschlagewerk zu wünschen ist, kann die Stellungnahme, vor allem in ihren zurückweisenden Passagen, keine Vorbildfunktion für den Umgang der Politik mit wissenschaftlicher Politikberatung für sich beanspruchen." (Stefan/Keil 1994: 6)

Im Hinblick auf das spezifische Thema der, wie sie titelt, "Unvereinbarkeit von Familie und Beruf" attestiert Notz (1994) dem Bericht, Familie "nicht ausschließlich als Ort der Geborgenheit, an dem der gestreßte (männliche) Berufsmensch Ruhe, Entspannung und liebevolle Zuwendung findet" zu behandeln. Doch beanstandet wird, es werde wie schon früher nicht problematisiert, daß durch die Tatsache, wonach "es den individuellen Aushandlungsprozessen der Partner überlassen bleibt, wer, wann, wie lange zu Hause bleibt, und wer das wie beruflich geregelt bekommt, weiterhin Frauen in bestimmte Rollen gezwängt werden. Sie füllen sie aus, weil ihnen nichts anderes übrig bleibt, wenn sie Kinder in ihre Lebensplanung einbezogen haben. Ein 'Familiengesetz', das die egalitäre Aufteilung der Familienarbeiten zwischen Männern und Frauen, die sich für diese Lebensform entschieden haben, wie wir es heute z.B. in Griechenland vorfinden... ist nicht zu erwarten und wird im Familienbericht nicht empfohlen" (372). Vielleicht, so schließt die Autorin, "brauchen wir keine Familienpolitik, sondern eine Menschenpolitik, die allen Menschen Lebensbedingungen bietet, 'die dazu beitragen, ein menschenwürdiges Dasein zu sichern'...". Das aber würde heißen, "daß Menschen nicht mehr als Anhängsel einer Familie, als 'Haupternährer' und 'Zuverdiener' betrachtet werden, sondern als eigenständige Individuen" (373).

Die Thematik der Vereinbarkeit von Familie und Beruf geht Ehinger (1995) unter Rückgriff auf das Konzept der strukturellen Rücksichtslosigkeit in 
rechtspolitischer Hinsicht an. "Nach Auffassung der Verfasserin produziert auch die Rechtsprechung strukturelle Ungerechtigkeiten in dem im Bericht definierten Sinne, z.B. in Trennungs- und Geschiedenenunterhaltsrecht, die überwunden werden sollten. Der Erwerbstätigenbonus und die Praxis, Einkünfte aus einer von den Ehegatten nicht geplanten oder nur wegen der Trennung oder Scheidung aufgenommenen Erwerbstätigkeit nicht dem bedarfsbestimmten Einkommen zuzurechnen, schmälern den Unterhaltsanspruch des kinderbetreuenden Elternteils und ist Beispiel für die Unterschätzung von Familienarbeit" (so der einleitende Kommentar der Redaktion).

Im einzelnen macht die Verfasserin geltend, bei der Auslegung unbestimmter Rechtsbegriffe, die der Gesetzgeber besonders häufig im Unterhaltsrecht verwendet hat, um Raum für mehr Einzelfallgerechtigkeit zu geben, habe sich ein Richterrecht entwickelt, das in Form von Leitlinien und Unterhaltstabellen der Oberlandesgerichte den juristischen Alltag in den Jugendämtern, Anwaltspraxen und Gerichten beherrsche. (10/11) In einer Wende zum Grundsätzlichen wird der Bezug zu Art. 3 Abs. 2 GG hergestellt, also zu der dem Staat aufgegebenen Förderung der tatsächlichen Durchsetzung der Gleichberechtigung. Angewandt auf die konkreten Beispiele heißt dies, daß für den Gesetzgeber nicht nur die Pflicht besteht, "die Gleichwertigkeit expressis verbis zu bestätigen, sondern sie auch rechtstechnisch umzusetzen, damit sie für die betroffenen Bürger erlebbar wird." $(13)^{17}$

Beachtenswert unter den uns hier interessierenden Gesichtspunkten sind zwei Aufsätze in der "Zeitschrift für Familienforschung" von Lewandowski (1996) und Netzler (1996), weil sie unter Bezug auf die Schlüsselkonzepte, "Humanvermögen" und "strukturelle Rücksichtslosigkeit" eine grundsätzliche Kritik an der damit versuchten Begründung von Familienpolitik üben. Dies bot 
F.-X. Kaufmann, der als stellvertretender Vorsitzender in der Kommission maßgeblich mitgewirkt hat, Anlaß zu einer ausführlichen Replik, in der er die theoretische Verankerung der Konzepte darlegt. Demnach geht der Begriff des Humanvermögens, der im Bericht "als Inbegriff aller gesellschaftlich nützlichen Kompetenzen der Bevölkerung" dient, auf Friedrich Lists "Theorie der produktiven Kräfte" zurück. Derjenige der "strukturellen Rücksichtslosigkeit" wurde "in Anlehnung an Vorstellungen aus der Gesellschaftstheorie Niklas Luhmanns und unter Bezugnahme auf die ökonomischen Konzepte 'soziale Kosten' und 'externe Effekte' formuliert" (Kaufmann 1997:9-11). ${ }^{18}$ Man sieht daran, daß die beiden Bezeichnungen nicht nur wegen ihrer Eingänglichkeit formuliert worden sind. Indessen ist es - so Kaufmann - die klare Absicht der Kommission gewesen, mittels dieser Begriffe die volkswirtschaftlich produktiven Leistungen der Familie ins öffentliche Bewußtsein zu heben (12). Damit ist die Rhetorik des Berichts angesprochen.

Kaufmann legt überdies dar, inwiefern eine Sozialberichterstattung von der Art, wie sie der Fünfte Familienbericht repräsentiert, nicht eigentlich unter Gesichtspunkten der Zweck-, sondern der Wertrationalität argumentiert, nämlich aus der bereits von Max Weber herausgearbeiteten Kulturbedeutung von Wissenschaft. Dieser Rückgriff auf die Weber'schen Kategorien ist bedenkenswert und erhellend, denn Max Weber war selbst durch seine Mitarbeit an der Landarbeiter-Enquête (1892-1899) und der Industriearbeiter-Enquête (1892-1912) an den Untersuchungen langjährig beteiligt, die, jedenfalls in einem weiteren Sinne, als Vorläufer der Sozialberichterstattung angesehen werden können.

17 Weitere Überlegungen zu juristischen Aspekten finden sich bei Grandke (1995)

18 Siehe hierzu auch die in Anm. 7 erwähnten Arbeiten von Krüsselberg und Lampert. 
Unter Bezug auf Habermas vertritt Kaufmann im weiteren die Auffassung, die den tragenden Konzepten zugrundeliegenden Erkenntnisinteressen der Wissenschaftler entsprächen nicht bloß der Dezision des einzelnen, sondern dieser würde sich auf Diskurse beziehen, "die entweder fachspezifisch oder politisch oder auf Kulturbedeutsamkeit hin fokussiert sind. Dabei ist uns allerdings nach vorherrschender wissenschaftsphilosophischer Auffassung eine Letztbegründung unmöglich. An ihre Stelle tritt im Idealfall der unendliche Diskurs unter den Prämissen von Gutwilligkeit und vernünftiger Argumentation; diese Prämissen sind in das Ethos moderner Wissenschaft eingebettet."

Zieht man an dieser Stelle eine erste Zwischenbilanz, läßt sich sagen: Der fünfte deutsche Familienbericht ist ein Dokument der Sozialberichterstattung, das sich interpretieren läßt als Beitrag zum öffentlichen Diskurs über Familie und Familienpolitik, mit der also in einem umfassenden Sinne eine "Definition der (gesellschaftlichen) Situation von Familie" angestrebt wird. Das ergibt sich aus dem Umfang und den inhaltlichen Schwerpunkten, der dualen (dialogischen) Grundstruktur, der Präsentation und den Adressaten, der rhetorischen Aura der Schlüsselkonzepte und entspricht überdies dem Selbstverständnis eines der Akteure. Gleichzeitig muß man feststellen, daß im konkreten Fall diese Intentionen sich nur beschränkt umsetzen ließen, sowohl hinsichtlich der unmittelbaren Rezeption im Parlament als auch in den Medien und der wissenschaftlichen Öffentlichkeit. Es besteht eine Diskrepanz zwischen Intention und Reaktion. Gilt das auch in einem (längeren) historischen Rückblick?

\section{Die Institutionalisierung der deutschen Familienberichte}

Der fünfte Familienbericht steht, die Numerierung zeigt es an, in einer Tradition. Sie signalisiert eine Institutionalisierung, die in den Entschließungen bzw. 
Beauftragungen des Bundestages ihre rechtliche Grundlage haben. ${ }^{19}$ Dabei wurde nach dem ersten Bericht, für den der Auftrag 1965 erteilt worden ist, und der unter Beizug von drei Wissenschaftlerinnen und vier Wissenschaftlern von Ministerien erstellt worden war, die Aufteilung in einen Sachverständigenbericht und eine Stellungnahme der Bundesregierung eingeführt. Überdies wurde festgelegt, jeder zweite Bericht solle sich mit der allgemeinen Situation von Familie befassen, also der erste, dritte und fünfte. Dazwischen handelt es sich um Berichte, in denen spezielle Bereiche vertieft dargestellt wurden (Leistungen der Familien im Erziehungs- und Bildungsprozeß, Situation der älteren Menschen in der Familie). Der in Auftrag gegebene sechste Bericht behandelt ausländische Familien in Deutschland. Ferner wurden in der Entschließung 1982 inhaltliche Themen vorgegeben, nämlich Familie und Arbeitswelt, Familie und Gesundheit sowie die Berücksichtigung familienpolitischer Elemente im Wohnungsbau. Gewissermaßen von selbst versteht sich seit jeher, daß die Berichterstattung die Aufarbeitung und Aktualisierung demographischer Daten beinhaltet. Schließlich wurde 1993 entschieden, es sei in künftigen Familien- und Jugendberichten die Situation der Kinder besonders in den Blick zu nehmen.

Offensichtlich war von Anfang an auch, daß die Berichte sich nicht nur auf eine Beschreibung der Situation von Familien beschränken sollten, sondern diese unter dem Gesichtspunkt der Notwendigkeit familienpolitischen Handelns beurteilen sollten, in erster Linie demjenigen des Staates. Dessen rechtliche Legitimation leitet sich in erster Linie von Artikel $6 \mathrm{GG}$ ab, doch sind auch weitere verfassungsrechtliche und gesetzliche Regelungen relevant für die Legitimation von und die Verpflichtung für Familienpolitik. In diesem Sinne ist

19 Für das Folgende siehe die Einleitung zum 5. Familienbericht (5: III). 
die Kennzeichnung "Definition der Situation" auch hinsichtlich der Doppeldeutigkeit des Begriffs der Definition angemessen. ${ }^{20}$

Die historische Kontinuität der Berichte, deren Rhythmus zwar, was die zeitlichen Abstände betrifft, nicht ganz regelmäßig war, legt nahe, nach Entwicklungen und Verschiebungen in den Inhalten $\mathrm{zu}$ fragen. Ebenso interessiert die Zusammensetzung der Sachverständigenkommission, nicht zuletzt angesichts ihrer institutionellen Unabhängigkeit (die durch die duale Grundstruktur eine formale Verstärkung erfuhr).

Geht man diesen Fragen nach, also jenen nach den Akteuren, stößt man auf folgende Sachverhalte: Vom zweiten (1975 erschienenen) bis fünften Familienbericht (1995) haben insgesamt 17 Persönlichkeiten als Sachverständige mitgewirkt. Zählt man die drei Expertinnen und vier Experten des ersten Berichtes mit, sind es 24 Persönlichkeiten. Bis auf eine Ausnahme (sowie eine damalige Privatdozentin) handelt es sich um Professorinnen und Professoren, hauptsächlich aus den Fächern Volkswirtschaftslehre, Bevölkerungswissenschaft, Haushaltswirtschaft, Rechtswissenschaft, Soziologie sowie Psychologie. ${ }^{21}$ Von diesen gehörten oder gehören zwei Drittel dem

20 Hier ist auch daran zu erinnern, dass der Begriff der Familienpolitik mindestens zwei Konnotationen haben kann. Die erste, die auch hier gemeint ist, bezeichnet Maßnahmen, Einrichtungen und weitere Aktivitäten, die gestützt auf rechtliche Grundlagen und/oder ordnungspolitische Vorgaben die Anerkennung und Förderung von "Familie" bezwecken (wobei damit - wie die Korrespondenz-These besagt - die "Definition" von Familie angesprochen wird). Diese Bedeutung entspricht weitgehend dem Englischen "policy". Familienpolitik kann aber auch den Charakter einer "Politik mit bzw. über Familie" haben, also der Auseinandersetzung darüber, welchen Zwecken "Familie" unterzuordnen ist, worin ihr Stellenwert besteht und in welchem Ausmaß ihr Anerkennung und Förderung zukommen. Hier handelt es sich um "politics". Diese beiden Konnotationen durchdringen sich insbesondere auch im deutschen Verständnis. - Im weiteren gehört zur FamilienpolitikAnalyse die Unterscheidung nach der Trägerschaft von familienbezogenen Maßnahmen, namentlich solcher staatlicher Art (auf unterschiedlichen Ebenen), nicht-staatlicher, verbandlicher Art sowie der Tätigkeit von "Selbsthilfe"-Organisationen, ausgehend von der Basisarbeit.

21 Zur Sichtweise und zum Selbstverständnis der Experten siehe Walter, 1993a. 
Wissenschaftlichen Beirat des Familienministeriums an. Diese Verflechtung wird zusätzlich verstärkt durch den Umstand, daß die beiden Personen, die im Ministerium für die Berichte zuständig waren, ihrerseits auch als Vertreter an den wissenschaftlichen Beratungen des Beirates teilnahmen.

Im weiteren haben sechs Persönlichkeiten an zwei oder drei Berichten mitgearbeitet. Sie, ebenso wie alle Vorsitzenden, gehören noch oder gehörten dem Beirat an. Das Sekretariat wurde seit dem zweiten Bericht vom DJI geführt; sein Direktor hat im Beirat den Status eines ständigen Gastes. Man kann also, seit 1968 der erste Bericht erstellt worden ist, eine offensichtliche personelle Kontinuität in der Familienberichterstattung feststellen, die überdies mit der Mitgliedschaft im Beirat korreliert.

Der Wissenschaftliche Beirat ist ein permanentes Beratungsorgan beim - nicht des Ministeriums. An seinen Sitzungen nimmt ständig mindestens ein Ministerialbeamter bzw. eine Ministerialbeamtin teil. Regelmäßig finden überdies Aussprachen mit Abteilungsleitern, Staatssekretär und bisweilen der Ministerin statt. Indessen ist der Beirat grundsätzlich frei, welche Themen er zum Gegenstand seiner Beratungen macht. Diese finden ihren Niederschlag in Gutachten und Stellungnahmen, deren Inhalt der Beirat in alleiniger Kompetenz verantwortet. Er verfügt übrigens, was für ein Beratungsgremium dieser Art bemerkenswert ist, über das Recht, die Mitglieder selbst vorzuschlagen. Die Sachverständigen für den Familienbericht werden hingegen vom Ministerium ausgewählt und berufen.

Vergleicht man nun die Themen der Beiratsgutachten und die Schwerpunkte in den Familienberichten, so ist offensichtlich, daß von Anfang an die Frage der Begründung von Familienpolitik und des Familienlastenausgleiches eine übergreifende Thematik bilden. Darüber hinaus kann man sagen, daß spezifische 
Themen, über die sich der Beirat gutachterlich geäußert hat, eine wichtige Rolle in den Familienberichten spielten.

Angesichts des Umstandes, daß der Beirat und weitere organisatorische Querverbindungen offensichtlich ein Pool für Mitglieder der Familienberichtskommission sind, sowie angesichts der inhaltlichen Durchdringung der Themen, scheint es mir darum gerechtfertigt, von einer Institutionalisierung zu sprechen, die nicht nur in der rechtlichen Verankerung und der zeitlichen Kontinuität manifest wird, sondern angesichts der Verbindung mit dem Beirat auch eine latente Komponente aufweist, die vor allem die Auswahl der Akteure betrifft. Vor diesem Hintergrund verdient die Entwicklung wichtiger Argumentationen besonderes Interesse.

\section{Entwicklungstendenzen vom ersten bis zum fünften Familienbericht}

Es liegen mittlerweile einige Aufsätze vor, in denen versucht wird, Linien der Darstellung und der Argumentation zu eruieren und darzustellen, so von Leu (1997), Walter (1993b), Lamm-Hess/Wehrspaun (1993) und Behning (1996). Sie stimmen nicht durchgängig überein; doch sie stellen im Hinblick auf die Themen, denen sie ihre Aufmerksamkeit schenken, Entwicklungen fest. Das hat selbstverständlich mit der im Laufe der letzten Jahrzehnte zu beobachtenden dreifachen Dynamik von Familie zu tun. Verändert haben sich die familialen Verhaltensweisen, die alltäglichen gesellschaftlichen Vorstellungen von Familie und die sozialwissenschaftlichen Perspektiven. Der Sozialwissenschaft obliegt es, Verhaltensweisen und Vorstellungen in den Blick zu nehmen und aufeinander zu beziehen, jedenfalls dann, wenn wissenssoziologische Reflexionen angestellt werden. Gewissermaßen der Kristallisationspunkt dieser drei Facetten ist die in den Berichten verwendete "Definition" von Familie und ihre "Verortung" in der Gesamtheit der privaten Lebensformen. Spiegelbildlich dazu - das läßt sich aus 
der einleitend erwähnten Korrespondenz-These ableiten - steht das Verständnis von Familienpolitik. Ich will kurz auf einzelne Punkte eingehen:

a) Die demographischen Daten drücken faktisch eine zunehmende Dokumentation und Anerkennung von Pluralität aus, obgleich gerade im fünften Familienbericht das Konzept als solches eher zurückhaltend verwendet wird. Mindestens so bedeutungsvoll ist indessen die vermehrte Heranziehung von Daten zusätzlich zu jenen der amtlichen Statistik, also die Ergebnisse breitangelegter, wiederholt durchgeführter Surveys sowie ganz allgemein die Befunde empirischer Untersuchungen. Dazu ist zu bemerken, daß die empirische Familienforschung in allen einschlägigen Disziplinen in den letzten Jahren einen großen Aufschwung erlebt hat. In der Einbeziehung dieser Literatur sehe ich das Bemühen um ein höheres Maß an Authentizität und den Versuch, (privaten) Sichtweisen von Familie gerecht zu werden. Dies drückt sich meines Erachtens überhaupt im Konzept der Pluralität aus. Damit einher geht eine Distanzierung von Vorstellungen der Normalität. ${ }^{22}$

Es lohnt sich, diesen Punkt wissenssoziologisch $\mathrm{zu}$ vertiefen, weil er möglicherweise nicht nur für die Familienberichterstattung relevant ist. Die amtliche Statistik verfügt bekanntlich traditionellerweise über eine gewisse "Definitionsmacht" hinsichtlich der von ihr untersuchten Phänomene. Sie hat ihren Grund in der gesetzlichen Verankerung der Datenerhebung, die häufig mit einer Auskunftspflicht einhergeht. Jedenfalls kommt den von ihr verwendeten Kategorien und den ermittelten Zahlen ein herausgehobener (quasimonopolitischer) Charakter zu. In jüngster Zeit wird er indessen durch

22 Die - erstaunlicherweise immer wieder aufflammende - Kontroverse, ob tatsächlich von einer Pluralisierung von Familienformen gesprochen werden kann, dürfte mit dem eingangs erwähnten normativen Gehalt des Begriffes Familie zusammenhängen. Sie lässt sich m.E. jedoch beilegen, wenn die unterschiedlichen faktischen und konzeptuellen Dimensionen für die Beschreibung familialer Lebensformen berücksichtigt werden. Für meine diesbezüglichen Vorschläge siehe Lüscher 1995b. 
andere Datenerhebungen relativiert, vorab durch die mittlerweile ebenfalls vergleichsmäßig durchgeführten Panelbefragungen. Auf wieder andere Weise trägt zu verschiedenen Themen auch die Demoskopie bei. Die aktuelle Sozialberichterstattung wird so zu einem wissenschaftlichen Feld, in dem jedenfalls hintergründig - Auseinandersetzungen zwischen verschiedenen Akteuren der makrosozialen Analyse ausgetragen werden. Inwiefern sind die (universitären) Wissenschaftler dabei Schiedsrichter, inwiefern Parteigänger?

b) Besonders markante Veränderungen gibt es - so Leu (1997) - in der Beurteilung der Erwerbstätigkeit der Frauen bzw. der Mütter. Diese wurde im ersten Familienbericht weitgehend unter negativen Gesichtspunkten gesehen, im zweiten und dritten widersprüchlich behandelt, wohingegen sich im fünften eine dezidiert andere Position abzeichnet. Sie drückt sich in der Auffassung aus, "eine einseitige und als ungerecht empfundene Verteilung der mit dem Familienleben verbundenen Belastungen stelle die außerordentlich wichtige Geschlechter- und Generationensolidarität in Frage." (Leu 1997: 84).

Lamm-Heß/Wehrspaun (1993) zeigen in ihrem im Rahmen der Konstanzer Untersuchungen entstandenen Vergleich zwischen dem dritten und dem vierten Familienbericht, daß letzterer eine wesentliche Offenheit für Alternativen gegenüber dem überkommenen Leitbild zeigt. Diese Linie wird dann im fünften Familienbericht fortgesetzt und mündet dort ein in die übergreifende Konzeption des Humanvermögens. Hinsichtlich der allgemeineren Thematik der im Familienbild enthaltenen Konstruktionen von Geschlecht in den Familienberichten stellt Behning (1996) vom ersten bis zum fünften Familienbericht ebenfalls eine unverkennbare Entwicklung zur Öffnung fest, in die sich allerdings der vierte Familienbericht nicht geradlinig einordnen läßt. 
c) Die Frage der Familiendefinition impliziert eine Auseinandersetzung mit dem Leitbild von Familie. Richtigerweise verweist Walter (1993b) - und unter Bezug auf ihn auch Leu - auf die besondere Bedeutung des bürgerlichen Modells. Daran messend stellt Walter im Blick auf die ersten drei Familienberichte zusammenfassend fest: "- (es) kristallisiert sich ein Kern dieses (in der Nachkriegszeit dominierenden) Familienverständnisses heraus, der in den Familienberichten bewahrt wird. Dieser ist zum einen in der festen Verbindung des Familienbegriffes mit dem der Ehe zu sehen. Zum andern wird durch die klare Polarität, die der Kernfamilie mit Mutter, Vater und Kindern vor allen anderen Formen gegeben wird, diese zum Modell oder Symbol für das institutionalisierte Familienverständnis überhaupt. - Daneben existiert ein Kranz von Prinzipien, die modifiziert, umformuliert oder kaum erwähnt werden: Haushaltsgemeinschaft, Vollständigkeitsprinzip und Abstammungsprinzip. ... Schließlich werden zwei Prinzipien unter dem maßgeblichen Einfluß der sozialwissenschaftlichen Begriffsbildung und Analyse neu bestimmt. Das Erziehungsprinzip wandelt sich zur Sozialisationsfunktion $\quad . .$, das Produktionsprinzip wird ... zu einer Funktion, namentlich der Generativen." Hinsichtlich der Geschlechtspolarität besteht gemäß Walter eine "ausgeprägte Vorstellung von Familie als einer Institution mit normativen Folgerungen bezüglich der angemessenen Mutterrolle..." (32/33). Im vierten Familienbericht erkennt Walter dann einen Neuansatz auf der Basis des Solidaritätsprinzips und eines veränderten Familienleitbildes. Was die politischen Zielsetzungen der Familienberichterstattung betrifft, kommt Walter zum Schluß, daß die Kommissionen für den zweiten und dritten Familienbericht offensichtlich ihre Arbeit darauf ausrichteten, "unterhalb des Familienleitbildes zu konkreten politischen Festlegungen zu kommen". Gleichzeitig stellt er fest, daß die beiden Positionen seiner Auffassung nach nicht in Einklang mit politisch dominanten Themen der Zeit standen, weil der zweite Bericht entgegen einer sich abzeichnenden Reformmüdigkeit ein "systematisches Familienpolitik-Programm 
auf wissenschaftlicher Grundlage formulierte", und der dritte, nach der Reform des Eherechts von 1977, "die traditionelle Aufgabenteilung zu rechtfertigen suchte". Walter sieht in beiden Fällen im Rückbezug auf das traditionelle Leitbild in der Familiendefinition einen Grund, weshalb die Berichte vergleichsweise folgenlos blieben. Seiner Ansicht nach zeigt das Beispiel des vierten Familienberichtes jedoch, "daß mit einer konkreten und auf ein familienpolitisches Ziel ausgerichteten Leitidee die Resonanz des Berichts im politischen Raum erhöht werden kann." (36) Die Leitidee liegt in der mit der Familiendefinition festgeschriebenen Solidarität der Generationen, die gemäß Walter eine "neuartige und konkrete Problemperspektive" darstellt (ebd.).

Leu (1995) vertritt die Auffassung, daß die Veränderungen vom ersten und zweiten bis zum vierten und fünften Familienbericht "bezüglich der Darstellung des Verhältnisses der Familie zum weiteren sozialen Kontext" sich zusammenfassen lassen als "Entwicklung von der Kritik eines Familismus mit Öffentlichkeitsdefiziten der Familie zur Formulierung von Familiendefiziten der Öffentlichkeit" (ebd. 84). Diesen Wandel "von einer gesellschaftlichen Defizitperspektive auf die Familie zu einer familienorientierten Defizitperspektive auf die Gesellschaft" stellt er besonders markant hinsichtlich des Themas Familie und Bildung fest. Er kommt zum Schluß, "daß mit der außerordentlich hohen Wertschätzung familialer Leistungen, die für den fünften Familienbericht kennzeichnend ist und die sich auch in der Familienorientierung seiner bildungspolitischen Vorschläge äußert, auch Veränderungen zentraler Merkmale des bürgerlichen Familienmodells verbunden sind" (60).

Eine zweite Zwischenbilanz ergibt: Die deutschen Familienberichte sind - wie erwähnt - sowohl in organisatorischer Hinsicht als auch angesichts ihrer Akteure in einem hohen Maße institutionalisiert. Unter diesen Umständen könnte man zunächst erwarten, daß sie eher beharrlich an überkommenen Leitbildern von 
Familie festhalten. Demgegenüber stimmen die vergleichenden Darstellungen weitgehend darin überein, daß sich markante Entwicklungen feststellen lassen. Diese reflektieren nicht nur die empirischen Veränderungen und den Wandel der alltäglichen Vorstellungen, sondern sie lassen sich als Ausdruck des Bemühens interpretieren, eine zunehmend eigenständige Argumentation zu entwickeln. Diese hat ihren Fokus in der Begründung von Familienpolitik und findet ihren vorläufigen - Ausdruck in der Konzeption, die im fünften Familienbericht dargelegt wird.

Ich bin der Auffassung, daß der hohe Grad der Institutionalisierung eine wichtige organisatorische Bedingung für die - wie ich sie bewerten möchte - hohe Qualität der nationalen deutschen Familienberichte ist. Im weiteren und unter inhaltlichen Gesichtspunkten meine ich, daß die Notwendigkeit der Auseinandersetzung mit Familienpolitik und die Möglichkeit, entsprechende Begründungen und Empfehlungen zu erarbeiten, mit anderen Worten, die aktive Auseinandersetzung mit Politikberatung, einen weiteren wichtigen Ansporn darstellt. Dieser ist seinerseits eng mit der dualen dialogischen Grundstruktur von Sachverständigenbericht und Stellungnahme der Regierung verknüpft, die einhergeht mit einer großen Unabhängigkeit der Experten und wiederum mit der Unabhängigkeit des Beirates zusammenhängt.

\section{Exkurs: Praktische Probleme der Dissimilation}

Aufgrund der starken Institutionalisierung der deutschen Familienberichte und ihrer Kontinuität lassen sie sich als Instrumente für die Zuteilung finanzieller Mittel und zur Beeinflussung der öffentlichen Diskurse über Familie und Familienpolitik verstehen. Doch die Daten über die Rezeption der Berichte legen den Schluß nahe, daß diese Absicht sich nur in einem vergleichsweise bescheidenen Maße realisieren läßt. Dies festzustellen scheint gerechtfertigt, 
auch wenn man in Rechnung stellt, daß bis jetzt die Rezeption nur teilweise empirisch erfaßt wurde.

Vollständigkeit dürfte überhaupt kaum möglich sein (was zur Charakterisierung von Rezeptionsprozessen dieser Art nicht ganz unwichtig ist). Es fehlen beispielsweise Daten darüber, wie häufig und wie intensiv die Berichte im Unterricht an Universitäten, Fachhochschulen und anderen mit Familien befaßten Ausbildungsstätten benutzt werden. Auch über die Zitationshäufigkeit in Lehrbüchern und Artikeln liegen bis jetzt keine Daten vor. Stichproben vermitteln allerdings nicht den Eindruck, die Berichte würden eine herausragende Rolle spielen, obgleich ihnen in den Rezensionen eine hohe Qualität der Dokumentation zugeschrieben wird. Diese zögerliche Rezeption hat meines Erachtens organisatorisch-praktische Gründe. Darauf möchte ich in diesem Exkurs kurz eingehen.

Ich beginne mit einem scheinbar nebensächlichen Detail: den Unsicherheiten des Bibliographierens und Zitierens der Berichte. Da es sich um eine Bundestagsdrucksache handelt, herausgegeben von der Bundesregierung, die Dokumente aber als "Familienberichte" bekannt werden, ist nicht klar, wie sie zitiert werden sollen, und vor allem geschieht dies auf unterschiedliche Weise. Dadurch vermindert sich die Prägnanz bzw. der Eindruck der Zitierhäufigkeit. Das Gleiche trifft auf die Gutachten des wissenschaftlichen Beirates zu, die ich als eine Art komplementäre Familienberichterstattung gekennzeichnet habe.

Was könnte über dieses Detail hinaus hinsichtlich der Organisation überdies getan werden, um die Rezeption der Berichte zu fördern? Das Ministerium selbst kümmert sich bis jetzt wenig um eine über die Medienorientierung hinausgehende Stützung der Diskurse. Die Veröffentlichung des Berichts wird faktisch zu einem Tagesereignis und damit hat es, was die aktive Orientierung 
der Öffentlichkeit betrifft, sein Bewenden. ${ }^{23}$ Hier könnte mehreres getan werden. Das Ministerium könnte beispielsweise Organisationen beauftragen, Symposien über die Berichte und Gutachten durchzuführen. Ebenso könnte sein Medienreferat zusammen mit Fachreferenten interessierten Vertretern der Medien vertiefende Orientierungen anbieten. Unsere Analysen der Tagespresse zeigen, daß es alles in allem eine relativ kleine Zahl von Journalistinnen und Journalisten sind, die sich in Deutschland intensiv mit Familienfragen beschäftigen. Auch Akademien, (Familien-) Verbände und wissenschaftliche Vereinigungen könnten aktiv werden.

Warum geschieht dies nicht häufiger? Auch hierfür gibt es m.E. einen trivialen praktischen Grund. Die Familienberichte (ähnlich wie andere Sozialberichte) erscheinen nämlich nicht zu einem klar vorhersehbaren Zeitpunkt. Das hängt u.a. mit der Terminierung und dem Verlauf der parlamentarischen Beratung zusammen. Ferner spielen politische und wahlstrategische Erwägungen eine Rolle. Organisationen und beispielsweise Akademien können in ihren - immerhin Monate im voraus zu erstellenden - Veranstaltungskalendern die Behandlung eines Familienberichtes nicht planen, ganz abgesehen davon, daß häufig erst beim Erscheinen eine breitere Öffentlichkeit und auch eine interessierte Fachöffentlichkeit tatsächlich erfahren, daß ein Bericht überhaupt vorliegt. Schließlich wird man kritisch feststellen müssen, daß die äußere Präsentation dieser Berichte nicht unbedingt geeignet ist, eine differenzierte Rezeption und eine differenzierte kritische Auseinandersetzung zu fördern.

\footnotetext{
${ }^{23}$ Löbliche Ausnahmen bilden die beiden Veranstaltungen, die nach der Veröffentlichung von zwei Gutachten des wissenschaftlichen Beirates ("Familie und Beratung", "Kinder und ihre Kindheit in Deutschland") unter organisatorischer Trägerschaft des Ministeriums durchgeführt werden konnten.
} 


\section{Familienberichte in anderen Ländern}

Familienberichte im engeren Sinne des Wortes gibt es - ich habe eingangs bereits darauf hingewiesen - auch in anderen europäischen Ländern. Sie weisen einzelne, jedoch nicht alle, der für die deutschen genannten konstitutiven Merkmale auf.

a) In Italien wurden bzw. werden von mehreren Organisationen Familienberichte erstellt (vgl. Donati 1989: 5-10). Diejenigen des CISF (Centro Internazionale Studi Famiglia) erscheinen seit 1989 in einem konsequent eingehaltenen Zweijahresrhythmus unter der gleichbleibenden Herausgeberschaft von P. Donati (1989, 1991, 1993, 1995, 1997). In der Einleitung zum ersten Bericht legt der Herausgeber die Abgrenzung zu den primär demographisch ausgerichteten Berichten des IRP dar, ebenso zu dem (zeitlich weiter zurückliegenden) Bericht der nationalen Kommission für Probleme der Familie (1983). Donatis Anliegen besteht darin, eine übergreifende theoretisch fundierte Evaluation der italienischen Familie zu bieten. Es gründet in seinem Verständnis von Familie als einem "autopoietischen System", das einher geht mit einer Fokussierung auf die beziehungssoziologische Analyse und ihren allgemeinen politischen Ausdruck in der Idee der "Nuova cittatinanza della famiglia" ("Family Citizenship") findet. ${ }^{24}$

Der erste Bericht behandelt mehrere der klassischen Themen der Familienanalyse wie Partnerschaft und Ehe, Familie, Kindheit und Adoleszenz, Familie und Frau, persönliche Dienstleistungen, Fragen der Ökonomie und des Rechtes. Die nachfolgenden Berichte stehen unter Schwerpunktthemen, wobei es sowohl im

24 "La società, vista ri-flessivamente dal punto di vista dei valori, delle strutture e dei compartimenti familiari, genera una forma generalizzata di famiglia che si può chiamare 'auto-poietica' in quanto si fa norma a se stessa e sfugge ('eccede') la società stessa." (Donati 1989:9) Darin drückt sich die Idee aus: "... la famiglia debba essere regolata nel senso del sostegno alla sua autonomia positivamente intesa, cioè nel quadro del bene comune. Entro questo orizzonte l'intento non è quello di dare soluzioni prefissate, ma è quello di offrire elementi e argomentazioni per un dibattito sereno e per decisioni responsabili" (ebd.). 
zweiten wie im vierten um die Generationenbeziehungen geht, im dritten um die Idee der "Cittadinanza" und im fünften um die Geschlechterbeziehungen. Diese Schwerpunktthemen werden in sieben bis neun Einzelkapiteln interdisziplinär abgehandelt, für die häufig Autorenteams verantwortlich zeichnen. Es handelt sich indessen nicht um eine Expertengruppe, die sich gemeinsam trifft und gemeinsam berät. Drei Berichte enthalten einen demographischen Anhang. Die publizistische Trägerschaft ebenso wie die Finanzierung der Arbeiten liegt beim CISF.

Die Federführung hat der Herausgeber inne. Dieser formuliert in Rahmenkapiteln die theoretischen Orientierungen. ${ }^{25}$ Diese italienischen Berichte haben also - im Unterschied $\mathrm{zu}$ den deutschen - eine nichtstaatliche Trägerschaft. Das Schwergewicht liegt bei theoretisch orientierten, allerdings empirisch ausführlich dokumentierten Darstellungen und Forschungsübersichten. Die familienpolitischen Implikationen werden eher beiläufig und im Rahmen einer allgemeinen Programmatik angesprochen.

b) In Österreich hat sich eine Familienberichterstattung in einem "Zehnjahres Rhythmus" entwickelt (1969, 1979, 1989 - in Vorbereitung 1999). Rein äußerlich gesehen bestand der Bericht von 1969 in einem Heft, derjenige von 1979 in sechs Teilheften und einer Zusammenfassung, jener von 1989 in einem eigentlichen Handbuch unter dem Titel "Lebenswelt und Familie", das als Nachschlagewerk über den aktuellen Stand der österreichischen und der internationalen Familienpolitik konzipiert worden ist. Dabei wurden auch ausführliche zeitdiagnostische Überlegungen angestellt, beispielsweise hinsichtlich einer intensiven Auseinandersetzung mit dem Konzept der "Postmoderne". Der Bericht 1989 wurde von einem Sachverständigengremium

25 Siehe in diesem Zusammenhang auch Donatis Beitrag zur Sitzung über Familienberichterstattung am Weltkongress für Soziologie in Bielefeld 1994 (Donati 1994). 
(Leitung H. Schattovits) verfaßt. Zusätzlich zur erwähnten ausführlichen Dokumentation enthält er auch einen Leistungsbericht über die Ergebnisse der Familienpolitik in Österreich, der dem Bericht vorangestellt wurde. Das Team der Sachverständigen konnte seinerzeit Folgerungen und einen Ausblick anfügen.

Die Vergabe des Berichtes von 1989 erfolgte auf ein Angebot des Koordinators, das Inhalt, Personen, Vorgehensweise, Zeitplan usw. umfaßte und die Basis für einen Werkvertrag bildete. Der Bericht wurde, gestützt auf die Beiträge der einzelnen, im Team erarbeitet. Es bestanden seitens des Ministeriums keinerlei Weisungen. - Für den Bericht von 1999 werden die Beiträge ebenso wie die Aufgabe der Koordination europaweit ausgeschrieben. ${ }^{26}$

Gemeinsamkeiten $\mathrm{zu}$ den deutschen Familienberichten bestehen in einer ausgeprägten und differenzierten sozialwissenschaftlichen Fundierung, die allerdings mehr enzyklopädischen Charakter hat und weniger die argumentative Entfaltung von Basisthemen beinhaltet, insbesondere nicht hinsichtlich der Begründung von Familienpolitik. Die rechtlich-institutionelle Regelung war bisher von Bericht zu Bericht eine andere. Ob über das offensichtlich im Gefolge des letzten Berichtes gegründete Österreichische Institut für Familienforschung eine (latente) Institutionalisierung zustande kommt, wird u.a. von den Erfahrungen mit dem Familienbericht 1999 abhängen.

c) In der Schweiz erschien 1978 ein verwaltungsinterner, im wesentlichen demographisch-deskriptiv gestalteter "Familienbericht". Im Anschluß daran wurde vom Eidgenössischen Departement des Innern eine Arbeitsgruppe eingesetzt, die zur Aufgabe hatte, den Stand der Familienpolitik darzustellen und 
Empfehlungen für deren Ausgestaltung zu machen. Dieser Bericht erschien 1982. Er hat offiziellen Charakter, wurde von der Regierung zur Kenntnis genommen und veröffentlicht. Er stellt insofern eine Besonderheit in der Landschaft der Familienberichterstattung dar, als er sich ausschließlich auf die Analyse von familienpolitischen Maßnahmen und Einrichtungen konzentriert, diese in einen historischen Kontext einordnet und für die wichtigsten Bereiche adressatenspezifische Empfehlungen aufstellt, so insbesondere hinsichtlich Familie und Arbeitswelt, Familie und Wohnen, Beratung und Elternbildung, Familie und elektronische Massenmedien (was zu jener Zeit noch kaum in der familienpolitischen Berichterstattung besprochen wurde), Besteuerung von Familie und Familienzulagen, Mutterschaftsschutz und Stipendienwesen. Der Bericht schließt mit Empfehlungen zum weiteren Ausbau der familienpolitischen Infrastruktur und mit dem Vorschlag, ein ständiges Organ für Familienpolitik einzurichten. Diese Empfehlungen wurden allerdings in der Folge nicht aufgenommen, was mit der grundsätzlich schwierigen Stellung von Familienpolitik in der Schweiz zusammenhängt (vgl. hierzu Lüscher 1982 bzw. 1988). Es liegt also keine Institutionalisierung der Familienberichterstattung vor. $\mathrm{Ob}$ sie sich in Verbindung mit einer 1995 eingerichteten Eidgenössischen Koordinationskommission für Familienfragen einstellt, wird sich in Zukunft erweisen müssen.

d) Schwierig zu übersehen scheint mir die neuere Entwicklung der französischen Familienberichterstattung. Hier könnte man wohl sagen, daß die Familienpolitik und die darauf bezogene permanente Analyse familialer Entwicklungen einen vergleichsweise hohen Grad der Institutionalisierung aufweist, mit der Folge, daß - zumindest in jüngster Zeit - keine integralen Familienberichte erstellt worden

\footnotetext{
${ }^{26}$ Diese Angaben für die österreichischen Familienberichte stützen sich, nebst den Dokumenten, auch auf persönliche Mitteilungen von H. Schattovits und L. Wilk. - Siehe auch Wild/Wintersberger 1996.
} 
sind. ${ }^{27}$ In anderen Ländern ist es umgekehrt die niedrige Spezifizierung und Institutionalisierung von Familienpolitik, die dazu führen, daß die Beschreibung familialer Entwicklungen in die allgemeine Sozialberichterstattung, namentlich demographischer Art, integriert ist. Die differenzierte vergleichende Darstellung von Inhalt, Trägerschaft und Adressaten steht m.W. noch aus. Für frühere Berichte liegt eine erste vergleichende Analyse von Lamm-Heß (1993) vor.

\section{Ausblick}

Der internationale Vergleich läßt die Merkmale der deutschen Familienberichterstattung nochmals deutlich hervortreten, die sich wie folgt zusammenfassen lassen:

- Es gibt eine rechtlich begründete manifeste Institutionalisierung und damit einhergehende Verfahrensregeln, einschließlich zeitliche Regelmäßigkeit.

- Es besteht eine thematische Kontinuität - im mehrfachen Sinne einer "Definition der (gesellschaftlichen) Situation" von Familie und ihrer Implikation für die Begründung und die Ausgestaltung von Familienpolitik.

- Die duale Form und die Verfahren der Unterrichtung des Parlamentes und der Veröffentlichung zeigen die Absicht, öffentliche Diskurse über Familie und Familienpolitik zu beeinflussen.

- Es läßt sich eine latente Institutionalisierung in der personellen und inhaltlichen Nähe zum Wissenschaftlichen Beirat sowie der Geschäftsführung der Sachverständigenkommission im Deutschen Jugendinstitut ausmachen.

27 Die Sozialberichterstattung über die Familie ist in das Gesamtsystem der Sozialberichterstattung integriert (pers. Mitt. von Jacques Commaille). Für eine aktuelle Darstellung des in verschiedener Hinsicht besonderen Verständnisses von Familienpolitik in Frankreich siehe Commaille/Martin 1998. 
Überdies ist hinsichtlich des weiteren Umfeldes erwähnenswert, daß die Familienberichte auf nationaler Ebene in einem gewissen Zusammenhang zu den Jugendberichten stehen, an denen das DJI einen noch stärkeren Anteil zu haben scheint als an den Familienberichten. Eine zusätzliche Klammer bildet die in jüngster Zeit festzustellende sozialpolitische Entdeckung der "Kindheit". Im weiteren ist auf die Familienberichte in einzelnen Bundesländern sowie in Kommunen hinzuweisen, wofür die nationalen Berichte häufig einen Bezug bilden. Schließlich gibt es Stellungnahmen zur Situation der Familie seitens anderer Träger, beispielsweise der Kirchen. - Wie vergleichen sich nun die Familienberichte mit anderen Formen der Sozialberichterstattung?

(1) Die deutschen Familienberichte repräsentieren eine Textgattung eigener Art; man könnte von einem Hybrid-Text sprechen. Einerseits haben sie einen systematischen wissenschaftlichen Teil; andererseits enthalten sie Ausführungen für die gesellschaftspolitische Praxis, nota bene sowohl im Sachverständigenteil als auch in der "Stellungnahme". Dabei ist es nun aber keineswegs so, daß die politischen Ausführungen lediglich Folgerungen oder Anwendungen der wissenschaftlichen Überlegungen sind. Vielmehr zeigt sich, daß die zentrale politische Fragestellung, nämlich die Begründung von Familienpolitik, systematische Rückwirkungen auf die wissenschaftliche Analyse hat, erkennbar etwa am zunehmend differenzierteren Verständnis familialer Leistungen und dem erfolgreichen Bemühen, die morphologische Pluralität prozessual zu vertiefen. Besonders deutlich kann man diese Verflechtung im Vergleich der fünf Berichte erkennen. Sie wird mit der latenten Institutionalisierung zusätzlich verstärkt. Dies weist darauf hin, daß die Wissenschaft wesentliche Impulse von der sozialpolitischen Praxis erfahren kann - der Einfluß also keineswegs nur einseitig gesehen werden sollte. 
(2) Obgleich die Familienberichte regelmäßig zu erstellen sind, handelt es sich keineswegs nur um Fortschreibungen. Sie sind nicht darauf eingerichtet, Trends $\mathrm{zu}$ ermitteln und sie sind, bei aller Kontinuität, nicht darauf festgelegt, stets dieselben Daten zu benutzen. Zwar spielen die Zahlen der amtlichen Statistik nach wie vor eine wichtige Rolle, und diese ist, wie man weiß, auf Kontinuität der Kategorien und Analysen ausgerichtet, hat also eine leicht konservierende, um nicht $\mathrm{zu}$ sagen, konservative Schlagseite. Doch die Familienberichtskommission konnte immer wieder neu an die Aufgabe herangehen, die gesellschaftliche Situation von Familie zu definieren, und sie konnte darum auch immer wieder neue Datensätze und Expertisen beiziehen. Auf diese Weise stellt sich eine gewisse Kontinuität höherer Ordnung ein, die eben zwangsläufig auf der Ebene der theoretischen Durchdringung der Materie angesiedelt ist.

(3) Eine derartige Theoretisierung der Sozialberichterstattung ergibt sich im Falle der Familienberichte auch aus ihrem Gegenstand. Ich meine damit die gewissermaßen konstitutive Unbestimmtheit des Begriffes der Familie als historische und als abstrakt-anthropologische, die sich - entsprechend der Korrespondenz-These - im Verständnis der Familienpolitik widerspiegelt. Mehr noch: sie findet sich auch im Recht und zwar - in Deutschland - im Grundgesetz. Man könnte sogar die Auffassung vertreten, die ebenso markante wie offene Formulierung von Art. $6 \mathrm{GG}$, in Verbindung mit anderen für die Familie relevanten Artikel, seien eine der tragenden Voraussetzungen der deutschen Familienberichterstattung - genauso, wie die Entscheidungen des BVerfG maßgebliche Richtlinien für den Familienlasten- und leistungssausgleich darstellen. Das wiederum hat zur Folge, daß die Familienberichte sich nicht auf die Darstellung quantifizierbarer Indikatoren der Entwicklung von Familie und Gesellschaft beschränken, sondern den qualitativen Aspekten der Bedeutung von Familie große Aufmerksamkeit schenken müssen. Dies stellt ein - m.E. noch zu 
wenig beachtetes - methodologisches Merkmal dar. Nicht übersehen sollte man auch, daß der Begriff des Risikos, der zentral für viele Sozialberichte ist, auf den Fall der Familie nicht oder nur mit einem zusätzlichen theoretischen Aufwand genutzt werden kann.

(4) Der diskurstheoretische Zugang, den ich zur Analyse der deutschen Familienberichte gewählt habe, hebt ihren rhetorischen Charakter hervor. Wiederum kann man sagen, das sei ein Merkmal des Gegenstandes. Doch es ist keineswegs so, daß nur Schlüsselbegriffe wie "Humanvermögen" und "Leistungsausgleich" in dieser Hinsicht wirken. Die wenigen Streiflichter auf die Presseberichterstattung erinnerten uns an die - tatsächliche oder vermeintliche Überzeugungskraft demographischer Zahlen. Sie besteht gewiß nicht nur für die Familienberichterstattung und ruft die ebenso plausible wie oft übersehene Tatsache in Erinnerung, daß wissenschaftliche Texte, namentlich solche, die sich an eine breitere Öffentlichkeit wenden, gewollt oder ungewollt rhetorische Elemente aufweisen. ${ }^{28}$ Werden solchermaßen die Texte der Sozialberichterstattung als "soziale Tatsachen" betrachtet, dann zeigt sich, daß ihre Form, ihre Rezeption und ihre Wirkung das Ergebnis enger Interdependenz von Inhalt, Akteuren, Rezeption und sozialem Kontext ist.

\section{Literatur ${ }^{29}$}

Andersson, B.-E., 1994: Public Policies and Early Childhood Education. European Early Childhood Education Research Journal 2, S. 19-32.

28 Diese These wird ausführlich im Projektbericht Bräuninger et al. 1996 begründet.

29 Angaben zu den Familienberichten und den Gutachten des wissenschaftlichen Beirates finden sich im Anhang. 
Behning, U., 1996: Zum Wandel des Bildes "der Familie" unter enthaltenen Konstruktionen von "Geschlecht" in den Familienberichten 1968-1993. Zeitschrift für Frauenforschung 14, S. 146-156.

Bericht über die Lage der Familie in der Schweiz (1978). Herausgegeben vom Eidgenössischen Departement des Innern. Bern.

Bertram, H., 1995: Zur Zukunft des Humanvermögens. Stimmen der Zeit 213, S. 555-563.

Bräuninger, B.; Lange, A., Lüscher, K., 1996: Familienwissenschaftliche Rhetorik. Konstanz: Forschungsschwerpunkt "Gesellschaft und Familie" (Arbeitspapier Nr. 20).

Commaille, J.; De Singly, F. (Hrsg.), 1997: The European Family. Dordrecht: Kluwer, S. 23-44.

Commaille, J.; Martin, C., 1998: Les enjeux politiques de la famille. Paris: Bayard Editions.

Deutscher Bundestag, 1994: 236. Sitzung, 24. Juni 1994. Bundestagsdrucksache 12, S. 20718-20737.

Donati, P., 1994: The Italian National Reports on the Family. ISA-Manuskript, ISA World Congress Bielefeld (mimeo).

Donati, P. (a cura di) Rapporti sulla famiglia in Italia, 1989, 1991, 1993, 1995, 1997. Cinisello Balsamo (Milano): Edizioni Paoline/San Paolo.

Ehinger, U., 1995: Der Begriff "strukturelle Rücksichtslosigkeiten" im fünften Familienbericht und seine Bedeutung für die Unterhaltsrechtsprechung. Familie, Partnerschaft, Recht 1, S. 10-13.

Grandke, A., 1995: Neue Herausforderungen an die Entwicklung des Familienrechts - Überlegungen aus der Sicht der neuen Bundesländer. Familie, Partnerschaft, Recht 1, S. 5-8. 
Kaufmann, F.-X., 1990: Zukunft der Familie. Stabilität, Stabilitätsrisiken und Wandel der familialen Lebensformen sowie ihre gesellschaftlichen und politischen Bedingungen. München: Beck.

Kaufmann, F.-X., 1997: Lässt sich Familienpolitik schlüssig begründen? Methodische Überlegungen zum fünften Familienbericht. Manuskript, Bielefeld, vervielfältigt.

Krüsselberg, H.-G., 1997: Ethik, Vermögen und Familie. Stuttgart: Lucius

Lamm-Heß, Y., 1993: Familienberichte als Spiegelbild nationaler Familienpolitik - Frankreich und Deutschland im Vergleich. Konstanz: Forschungsschwerpunkt "Gesellschaft und Familie" (Arbeitspapier Nr. 7).

Lamm-Hess, Y.;Wehrspaun, C., 1993: Frauen- und Müttererwerbstätigkeit im Dritten und Vierten Familienbericht. Konstanz: Forschungsschwerpunkt "Gesellschaft und Familie" (Arbeitspapier Nr. 4).

Lampert, H., 1993: Wer "produziert" das Humanvermögen e4iner Gesellschaft. In: Glatzel, N.; Kleindienst, E. (Hrsg.) Die personale Struktur des gesellschaftlichen Lebens. Berlin: Duncker \& Humblot.

Lampert, H., 1996: Priorität für die Familie. Berlin: Duncker \& Humblot.

Lange, A. et al., 1999 in Vorbereitung: Wandel von Kindheit - Wandel von Familie: Zur rhetorik sozialwissenschaftlicher Texte. Österreichische Zeitschrift für Soziologie.

Leu, H. R., 1995: Von der Bildungsorientierung der Familie zur Familienorientierung des Bildungssystems. Familie und Bildung im Spiegel der Familienberichte der Bundesregierung. Recht der Jugend und des Bildungswesens 43, S. 48-60. 
Leu, H. R., 1997: Die Familienberichte der Bundesregierung. In: Noll, H.-H. (Hrsg.). Sozialberichterstattung in Deutschland. Weinheim: Juventa, S. 73-92.

Lewandowski, S., 1996: Rational und defizitär? Zur Bestimmung eines familienpolitischen Grenznutzens - Eine Kritik am fünften Familienbericht. Zeitschrift für Familienforschung 8, S. 43-51.

Lüscher, K., 1982: Familienpolitik - Realität oder Utopie. Die Anfänge schweizerischer Familienpolitik. Pro Familia, S. 1-21.

Lüscher, K., 1988: Familienpolitik im liberalen Bundesstaat: Das Beispiel der Schweiz. In: Höhn, C.; Linke, W.; Mackensen, R. (Hrsg.). Demographie in der Bundesrepublik Deutschland: Festschrift für Karl Schwarz. Schriftenreihe des Bundesinstituts für Bevölkerungsforschung. Boppard: Boldt, S. 207-217.

Lüscher, K., 1995a: "Was heißt heute Familie? Thesen zur Familienrhetorik." In: Gerhardt, U.; Hradil, S.; Lucke, D.; Nauck B. (Hrsg.) Familie der Zukunft. Lebensbedingungen und Lebensform. Opladen: Leske und Budrich.

Lüscher, K., 1995b: "Postmoderne Herausforderungen der Familie". In: Familiendynamik, Heft 3. Stuttgart: Klett-Cotta, S. 233-251.

Lüscher, K., 1997: Familienleitbilder und Familienpolitik. In: Meier, U. (Hrsg.). Vom Oikos zum modernen Dienstleistungshaushalt. Festschrift für Rosemarie von Schweitzer. Frankfurt: Campus Verlag, S. 381-397.

Netzler, A.., 1996: Anmerkungen zu "Rational oder defizitär"? Zeitschrift für Familienforschung 8, S. 52-58.

Noll, H.H. (Hrsg.), 1997: Sozialberichterstattung in Deutschland. Weinheim: Juventa. 
Notz, G., 1994: Fünfter Familienbericht Familie und Erwerbsarbeit - die Unvereinbarkeit von Familie und Beruf. Theorie und Praxis der sozialen Arbeit, S. 370-373.

Rothenbacher, F., 1997: Familienberichterstattung in und für Europa. In: Noll, H.-H. (Hrsg.). Sozialberichterstattung in Deutschland. Weinheim: Juventa, S. 93-124.

Schütz, W. I. J., 1997: Deutsche Tagespresse 1997. In: Mediaperspektive, 12, 97, S. 663-684.

Stefan, A.; Keil, S., 1994: Familien und Familienpolitik im geeinten Deutschland. Zukunft des Humanvermögens. Familienpolitische Informationen EAF 33, S. 2-6.

Walter, W., 1993a: "Ich bin nur mäßig enttäuscht darüber." Zur Interpretation der Familienberichterstattung und der Sachverständigen-Rolle im Lichte von Experteninterviews. Konstanz: Forschungsschwerpunkt "Gesellschaft und Familie". (Arbeitspapier Nr. 1).

Walter, W., 1993b: Vom Familienleitbild zur Familiendefinition. Familienberichte und die Entwicklung des familienpolitischen Diskurses. Konstanz: Forschungsschwerpunkt "Gesellschaft und Familie". (Arbeitspapier Nr. 5).

Wilk, L.; Wintersberger, H., 1996: Paradigmenwechsel in Kindheitsforschung und -politik. Das Beispiel Österreich. In: Zeiher, H.; Büchner, P.; Zinnecker, J. (Hrsg.). Kinder als Außenseiter? Weinheim: Juventa, S. 29-55.

Zeitschrift für Soziologie, 1976: Besprechungsforum Zweiter Familienbericht. Mit Beiträgen von K. Lüscher, K. Grossmann, W. Albers und F.X. Kaufmann, S. 285-306. 



\section{Anhang}

\subsection{Eckdaten der Familienberichte der Bundesregierung}

\begin{tabular}{|l|c|c|c|c|c|}
\hline & 1. Bericht & 2. Bericht & 3. Bericht & 4. Bericht & 5. Bericht \\
\hline $\begin{array}{l}\text { Berufung der Kom- } \\
\text { mission }\end{array}$ & 23.06 .65 & 31.07 .70 & 19.12 .75 & 12.09 .83 & 06.03 .91 \\
\hline Berichtsauftrag & $\begin{array}{c}\text { Lage der Familie in } \\
\text { der Bundesrepublik } \\
\text { Deutschland (BT- } \\
\text { Beschluß) }\end{array}$ & $\begin{array}{c}\text { Leistungen und } \\
\text { Leistungsgrenzen } \\
\text { der Familie hin- } \\
\text { sichtlich des erzie- } \\
\text { hungs- und Bil- } \\
\text { dungsprozesses der } \\
\text { jüngeren Generation }\end{array}$ & $\begin{array}{c}\text { Grundfunktionen } \\
\text { (Aufgaben) der } \\
\text { Familie }\end{array}$ & $\begin{array}{c}\text { Situation der Familie } \\
\text { mit alten Menschen; } \\
\text { Situation der alten } \\
\text { Menschen im Hinblick } \\
\text { auf die Familie }\end{array}$ & $\begin{array}{c}\text { Umfassende Dar- } \\
\text { stellung der Situa- } \\
\text { tion der Familien } \\
\text { unter besonderer } \\
\text { Berücksichtigung } \\
\text { der Lage der Fa- } \\
\text { milien in den neuen } \\
\text { Bundesländern }\end{array}$ \\
\hline $\begin{array}{l}\text { Konstituierende } \\
\text { Sitzung }\end{array}$ & - & 05.11 .70 & - & 02.11 .83 & 02.05 .91 \\
\hline $\begin{array}{l}\text { Fertigstellung des } \\
\text { Kommissionsberichts }\end{array}$ & - & $03 . / 03.05 .74$ & 01.07 .78 & 06.12 .85 & 28.10 .93 \\
\hline $\begin{array}{l}\text { Fertigstellung: } \\
\text { Bericht und } \\
\text { Stellungnahme }\end{array}$ & 25.01 .68 (integriert) & 15.04 .75 & 20.08 .79 & 13.10 .86 & 15.06 .94 \\
\hline
\end{tabular}

Quellen: Leu 1997, Walter 1993 


\subsection{Gutachten des wissenschaftlichen Beirates für Familienfragen beim "Bundesfamilienministerium"}

\begin{tabular}{|c|c|c|c|}
\hline Gutachten & Jahr & Vorsitzender $^{3)}$ & Ministerin $^{3}$ \\
\hline Zur Reform des Familienlastenausgleichs & 1971 & Albers & Strobel \\
\hline Familie und Wohnen & 1975 & Kaufmann & Focke \\
\hline Leistungen für die nachwachsende Generation & 1979 & Krüsselberg & Huber \\
\hline Familien mit Kleinkindern & 1980 & Krüsselberg & Huber \\
\hline Familie und Arbeitswelt & 1984 & Krüsselberg & Geissler \\
\hline Familie und Medien & 1984 & Krüsselberg & Geissler \\
\hline Familienpolitik nach der Steuerreform & 1988 & Willeke & Süssmuth \\
\hline $\begin{array}{l}\text { Erziehungsgeld, Erziehungsurlaub und die An- } \\
\text { rechnung von Erziehungszeiten in der Renten- } \\
\text { versicherung }\end{array}$ & 1989 & Willeke & Süssmuth \\
\hline $\begin{array}{l}\text { Leitsätze und Empfehlungen zur Familienpolitik } \\
\text { im vereinigten Deutschland }\end{array}$ & 1991 & Keil & Rönsch \\
\hline $\begin{array}{l}\text { Zur Berechnung des steuerfreien Existenz- } \\
\text { minimums für den Lebensunterhalt eines Kindes }\end{array}$ & 1992 & Keil & Rönsch \\
\hline Familie und Beratung & 1993 & Keil & Rönsch \\
\hline $\begin{array}{l}\text { Zur Weiterentwicklung des Familienlastenaus- } \\
\text { gleichs nach den Entscheidungen des Bundesver- } \\
\text { fassungsgerichts seit } 1990\end{array}$ & 1994 & Liegle & Nolte \\
\hline $\begin{array}{l}\text { Kinder und ihre Kindheit in Deutschland. - Eine } \\
\text { Politik für Kinder im Kontext von Familienpolitik }\end{array}$ & 1997 & Liegle & Nolte \\
\hline
\end{tabular}




\subsection{Inhaltsverzeichnisse der deutschen und der italienischen Familienberichte}

\section{A. Familienberichte der Bundesregierung}

1. Bericht 1968 (Bundestagsdrucksache V/2532)

A. Demographische und sozio-kulturelle Strukturen und Tendenzen

I. Der Wachstumsprozeß der Bevölkerung

II Entstehung und Entwicklung der Familien in der Gegenwart

III Entwicklung der Rollenstruktur und Autoritätsverhältnisse der Ehegatten

IV Die Stabilität der Ehen und Familien

V außerhäusliche Erwerbstätigkeit der Mütter von noch nicht schulpflichtigen und schulpflichtigen Kindern

VI Entwicklung des Freizeitverhaltens der Familien

VII Familie und weiterführender Schulbesuch der Kinder

VIII Das Verhältnis der Familie zum öffentlichen Raum

B. Die ökonomischen Lebensbedingungen der Familie

I Einkommenslage

II Wohnverhältnisse

III Eigentums- und Vermögensbildung

2. Bericht 1975 (Bundestagsdrucksache 7/3502)

Stellungnahme der Bundesregierung
A. Einleitung
B. Grundzüge der Familienpolitik der Bundesregierung
C. Stellungnahme zu Vorschlägen der Kommission
I. Einkommenspolitische Maßnahmen
II. Stärkung der Erziehungsfähigkeit der Eltern 
III. Hilfen für frühkindliche Erziehung

IV. Förderung der Elementarerziehung

V. Familie und Wohnen

VI. Familie und Freizeit

VII. Familie und ländlicher Raum

VIII. Politik für benachteiligte Familien

D. Schlußbemerkungen

Bericht der Sachverständigenkommission
A. Familie und Sozialisation
I. Einleitung
II. Familie und Elternschaft: Gesellschaftliche Bedingungen kindlicher Sozialisation

III. Kommunikationsstrukturen und -probleme in der Familie

IV. Sozialisationswirkungen der Familie

V. Problemfelder und Problembedingungen familialer Sozialisation

B. Familienpolitische Maßnahmen

VI. Zielvorstellungen und Organisationsformen von Familienpolitik

VII. Maßnahmen zur materiellen Sicherung familialer Sozialisation

VIII. Familienbezogene Wohnungs- und Siedlungspolitik

IX. Pädagogisch-soziale Einwirkungen auf das familiale Erziehungsmilieu des Kindes

X. Familienergänzende und -ersetzende Institutionen

XI. Zusammenfassung und Vorschläge der Kommission

C. Anhang

3. Bericht 1979 (Bundestagsdrucksache 8/3121) 
Stellungnahme der Bundesregierung

Bericht der Sachverständigenkommission

1. Einleitung

2. Definition und Aufgaben der Familie

3. Sozialökonomische Lage der Familien

4. Familie und Plazierung

5. Familie und Bevölkerung

6. Leistungen der öffentlichen Hand für Familien

7. Exkurs: Ausländische Familien in der Bundesrepublik

8. Schlußfolgerungen

4. Bericht 1986 (Bundestagsdrucksache 10/6145)

Stellungnahme der Bundesregierung

1. Rechtsgrundlage/Auftrag

2. Familien- und altenpolitische Grundsätze der Bundesregierung

3. Bereiche besonderer politischer Aufmerksamkeit beim Zusammenleben der Generationen

4. Schlußbemerkungen

Bericht der Sachverständigenkommission

1. Einleitung

2. Kurzgefaßte Berichtsergebnisse

3. Struktureller Wandel in Bevölkerung, Familie, Wohlfahrtsentwicklung und Erwerbsleben

4. Älterwerden in unserer Zeit 
5. Die Lebenssituation der älteren Menschen und ihrer Familien nach Jahrgangsgruppen

6. Familiäre Beziehungen zwischen den Generationen

7. Lebensraum der älteren Menschen und der Familien - Wohnung und Siedlung

8. Soziale Infrastruktur für ältere Menschen und Familien

9. Der ältere Mensch und die Familie aus der Sicht der Medizin

10. Konfliktlagen, die (familien)politisches Handeln verlangen

11. Familienpolitische Forderungen der Kommission für den Vierten Familienbericht

5. Bericht 1994 (Bundestagsdrucksache 12/7560)

Stellungnahme der Bundesregierung

1. Stellenwert und Funktion der Familie in der Bundesrepublik Deutschland

2. Familie im Wandel

3. Erfordernisse und Maßnahmen in Politik und Gesellschaft

4. Schlußbemerkung

Bericht der Sachverständigenkommission

I. Familie und Gesellschaft

II. Grunddaten zu Bevölkerung, Familien und Privathaushalten

III. Berichtsergebnisse im Überblick

IV. Wandel des innerfamilialen Zusammenlebens

V. Familienrecht im geeinten Deutschland

VI. Lebenslagen der Familien in den alten und neuen Bundesländern

VII. Familie und Erwerbsarbeit

VIII. Stützungsnetze für Familien

IX. Familie und Bildung - Zur Familienorientierung des Bildungswesens 
X. Familie und Gesundheit

XI. Aktuelle Aufgaben der Familienpolitik: Der familienpolitische Handlungsbedarf

XII. Schutz und Förderung der Familie als politischer Auftrag

\section{B. Italienische Familienberichte CISF (Herausgeber: Pierpaolo Donati)}

Primo rapporto (1989):

I L'emergere della famiglia "auto-poietica" (P. Donati)

II La cultura della famiglia esplorata dai sondaggi demoscopici (G. Calvi)

III Le problematiche della coppia e del matrimonio (E. Scabini e V. Cigoli)

IV Famiglia, infanzia, adolescenza (P. Di Nicola)

V Famiglia e donna (B. Barbero Avanzini)

VI Famiglia e servizi sociali personali (G. Rossi Sciumè)

VII Famiglia e sistema economico (G. De Rita e C. Collicelli)

VIII Innovazioni legislative e giurisprudenziali sulla famiglia (O. Fumagalli Cerulli)

Secondo rapporto (1991):

I Equità generazionale: un nuovo confronto sulla qualità familiare (P. Donati)

II Contributo delle generazioni ai cambiamenti recenti nei comportamenti e nelle forme familiari (P. De Sandre)

III Il problema della denatalità in Italia: intensità, conseguenze, proposte di intervento (G. C. Blangiardo)

IV La condizione dell'infanzia (G. B. Sgritta)

$\mathrm{V}$ Le famiglie monogenitoriali in Italia (G. Rossi Sciumè, E. Scabini)

VI L'impatto sociale delle tecnologie di riproduzione (S. Spinsanti)

VII Famiglia e politiche sociali in Italia (C. Collicelli) 
VIII Famiglia e comunità ecclesiale (G. Fregni)

IX La promozione sociale della famiglia: proposte legislative ed esperienze di servizi innovativi

Terzo rapporto (1993):

I Le nuove mediazioni familiari: Le "relazioni invisibili" portatrici di nuovi diritti di cittadinanza (P. Donati)

II Il ruolo e le prospettive della famiglia nelle rappresentazioni degli Italiani (Risultati di un'indagine campionaria nazionale realizzata da Eurisko)

III Famiglia e cultura degli affetti (C. Pontalti)

IV Famiglia, mass media e televisione (F. Casetti, M. Comand)

$\mathrm{V}$ I nessi tra famiglia e scuola e l'associazionismo familiare in campo scolastico (L. Corradini)

VI Viaggo fra le contraddizioni del comportamento riproduttivo: dal rifiuto del figlio alla sua ricerca "A tutti i costi" (G. C. Blangiardo, G. Rossi)

VII Famiglie e comunità terapeuriche (M. Cagossi)

VIII La famiglia come soggetto di mediazione nei processi migratori (C. Collicelli)

IX La famiglia come mediatore dei comportamenti economici individuali (G. Malerba)

X La famiglia nell'esperienza giuridica tra mediazione e soggettività (G. Garancini)

XI Sintesi finale: Indicazioni operative (P. Donati)

Quarto rapporto (1995)

I Il malessere generazionale della famiglia: Dove va l'intreccio fra le generazioni? ( P. Donati)

II Cambiamenti familiare e relazioni generazionali: una lettura demografica (A. Golini, A. Silvestrini) 
III Rappresentazioni e comportamenti della famiglia: tre livelli generazionali a confronto (G. Calvi, Ch. Lundari)

IV Fra le generazioni modelli di connessione simbolica (V. Padiglione, C. Pontalti)

V La famiglia con adolescenti: uno snodo critico inter-generazionale (E. Scabini, E. Marta)

VI Generazioni di genitori e generazioni di figli: mondi incomunicabili? (P. Di Nicola, M. de Bernat)

VII Varianti dis-generazionali oggi: Fra simbolico e pre-simbolico (L. Ancona)

VIII Famiglia e devianza giovanile (C. Collicelli)

IX Diritto e relazioni inter-generazionali (A. C. Moro)

Quinto rapporto (1997): Uomo e donna in famiglia. Differenze, ruoli, responsabilità

Presentazioni: Famiglia e gender: Fra omogeneizzazione e nuove differenziazioni

I La famiglia come relazione di gender: morfogenesi e nuove strategie (P. Donati)

II Eventi demografici e differenze di genere lungo il ciclo di vita familiare (G. C. Blangiardo)

III Uomo e donna nei processi di trasformazione della famiglia in Italia (C. Collicelli)

IV Uomo e donna di fronte al percorso matrimoniale, alla separazione e al divorzio: aspetti psicologici e sociali (E. Scabini, R. Iafrate)

$\mathrm{V}$ Le differenze di genere presso i bambini e i giovani dalle ricerche campionarie nazionali realizzate da eurisko (G. Calvi-Parisetti, M. Lucchini)

VI Dinamiche psicopatologiche delle relazioni familiari con riferimento alle loro dimensioni sessuate (G. Vella, C. Loriedo, D. Solfaroli Camillocci) 
VII Il genere maschile e femminile nel magistero pontifico sulla famiglia e nella prassi della pastorale familiare (G. Fregni)

Conclusioni: The gendering of...: Dare un genere alla famiglia alle sur identità, alle sur relazioni 


\section{Forschungsschwerpunkt „Gesellschaft und Familie“6}

Aus Anlaß des zehnjährigen Bestehens des Forschungsschwerpunkts „Gesellschaft und Familie“ ist - als Arbeitspapier Nr. 30 - im Juni 1999 ein Tätigkeitsbericht erschienen, der eine kommentierte Bibliographie aller Publikationen enthält. Er kann bei folgender Adresse bezogen werden:

Prof. Dr. Kurt Lüscher

Universität Konstanz, Sozialwissenschaftliche Fakultät, FG Soziologie, Fach $<$ D33 $>$, D-78457 Konstanz

Tel: 07531/88-2670/2671, Fax: 07531/88-3038

E-mail: Kurt.Luescher@uni-konstanz.de

\section{Zuletzt erschienene Arbeitspapiere}

Nr. 25.1: Jutta Eckert-Schirmer: Einbahnstraße Pflegefamilie? Zur (Un)Bedeutung fachlicher Konzepte in der Pflegekinderarbeit. März 1997.

Nr. 25.2: Frank Ziegler: Jugendamtliche Handlungsmuster und das Zustandekommen von Besuchskontakten in Pflegekindschaftsverhältnissen. März 1997.

Nr. 25.3: Hans J. Hoch: Vormundschaftsgericht und Pflegekindschaft ( $§ 33$ KJHG). Die richterliche Regulation von Pflegekindschaftsverhältnissen und ihre Verknüpfung mit dem jugendamtlichen Verfahren. März 1997.

Nr. 26: Bettina Bräuninger, Andreas Lange und Kurt Lüscher: „Krieg zwischen den Generationen“? Die Darstellung von Generationenbeziehungen in ausgewählten Sachbuchtexten. Juli 1997.

Nr. 27: Andreas Lange und Kurt Lüscher: Kinder und ihre „Medienökologie“. Eine Zwischenbilanz der Forschung unter besonderer Berücksichtigung des Leitmediums Fernsehen. Oktober 1997.

Nr. 28: Kurt Lüscher und Karl Pillemer: Intergenerational Ambivalence. A New Approach to the Study of Parent-Child Relations in Later Life. Oktober 1997. 
Nr. 29: Kurt Lüscher: A Heuristic Model for the Study of Intergenerational Ambivalence. Juli 1998.

Nr. 30: Kurt Lüscher: Die Bedeutungsvielfalt von Familie. Zehn Jahre Forschungsschwerpunkt "Gesellschaft und Familie" an der Universität Konstanz. Juni 1999

Nr. 31: Andreas Lange, Frank Lettke und Frank Ziegler: Forschung über Jung und Alt für Jung und Alt. Mai 1999. 